\title{
Bifurcations and instabilities in rotating, two-layer fluids: II. $\beta$-plane
}

\author{
A. F. Lovegrove ${ }^{1, *}$, I. M. Moroz ${ }^{2}$, and P. L. $\operatorname{Read}^{1}$ \\ ${ }^{1}$ Atmospheric, Oceanic and Planetary Physics, University of Oxford, United Kingdom \\ ${ }^{2}$ Mathematical Institute, University of Oxford, United Kingdom \\ *Present address: Detica Ltd., Guildford, United Kingdom
}

Received: 20 April 2001 - Revised: 16 October 2001 - Accepted: 29 November 2001

\begin{abstract}
In this paper, we show that the behavior of weakly nonlinear waves in a 2-layer model of baroclinic instability on a $\beta$-plane with varying viscosity is determined by a single, degenerate codimension three bifurcation. In the process, we show how previous studies, using the method of multiple scales to derive evolution equations for the slowly varying amplitude of the growing wave, arise as special limits of the general evolution description.
\end{abstract}

\section{Introduction}

In a recent paper (Lovegrove et al. (2001), hereafter denoted by I), the spectral amplitude equations for the twolayer model of baroclinic instability on an $f$-plane were used to examine the differences between the multiple-scales approximations, developed by Pedlosky $(1970,1971)$ and Moroz (1981) for different dissipative regimes. This was possible because the derivation of the spectral amplitude equations does not require any restriction to be placed on the size of the dissipation parameter, $r$, thereby giving the spectral amplitude equations validity over the range $0 \leq r \leq 1$.

The equations were shown to reproduce the behavior observed in both the strongly and weakly dissipative limits when the spectral variables $x$ and $y$ were restricted to being real. It was then shown that, because the complex spectral amplitude equations were equivariant under the action of the $O(2)$ symmetry group, the results from the real system could be generalised to the complex case. It was also shown that the strongly dissipative multiple-scales approximation arises naturally from the weakly dissipative multiple-scales equations. It was found that the strong dissipation limit was valid for $r>0.07$, while the weak dissipation limit holds for $0<r<0.03$.

The bifurcations present in the weakly dissipative multiple-scales approximation were shown to originate in a degenerate bifurcation on the inviscid axis, and that close to

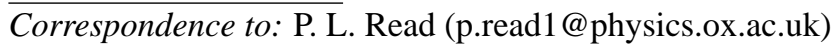

this bifurcation, fourth-order terms in the normal form expansion may be ignored. Small $O(2)$ symmetry-preserving perturbations to this third-order normal form then give rise to the Lorenz equations (Moroz, 1981). Further away from the degenerate bifurcation, fourth-order terms in the normal form expansion become important, corresponding to a transition from weakly to strongly dissipative behavior. A subset of the symmetry-preserving perturbations was then shown to produce the equations discovered by Pedlosky (1970) in his multiple-scales investigation of the inviscid system.

In this paper, we reinstate $\beta$ and develop a unified picture of bifurcations of weakly-nonlinear waves in a two-layer model of baroclinic instability with varying viscosity. Section 2 summarizes multiple-scales analyses for both strongly and weakly dissipative systems on the $\beta$-plane. In Sect. 3, the spectral amplitude equations are presented in both the original and co-rotating frames, and the bifurcations obtained with strong and weak dissipation are analysed in Sect. 4. Section 5 considers the circle map appropriate for the $\beta$-plane model. Section 6 considers the consequences of increasing $\beta$ in the spectral equations, while Sect. 7 discusses the inviscid, weakly-dispersive limit. Finally, we summarize and discuss our results in Sect. 8.

\section{Multiple scales analyses}

\subsection{Strongly dissipative systems: $r=O(1)$}

Romea (1977) and Moroz (1981) both performed multiplescales expansions of the two-layer quasi-geostrophic equations for a two-layer model on a $\beta$-plane, in which the dissipation was strong. Using the supercriticality parameter, $\mathcal{F}$, as the small parameter in the expansion, where

$F-F_{c}=\mathcal{F} \quad$ with $\quad \mathcal{F} \ll \mathrm{F}_{\mathrm{c}}$,

the critical value of the Froude number, $F_{c}$, at which the flow 
becomes unstable is given by

$F_{c}=\frac{K^{2}}{2}+\frac{1}{2 U_{d}^{2}}\left(\frac{\beta^{2} F_{c}^{2}}{K^{2}\left(K^{2}+F_{c}^{2}\right)^{2}}+\frac{r^{2} K^{2}}{k_{n}^{2}}\right)$,

while the wave speed is

$c_{r}=U_{s}-\frac{\beta}{\left(K^{2}+F_{c}\right)}$,

where $U_{s}=\left(u_{1}+u_{2}\right) / 2$.

In contrast to the $f$-plane model discussed by Lovegrove et al. (2001), the wave speed on the $\beta$-plane now depends on the wavelength. The wave no longer travels at the speed of the mean-flow, but the presence of the planetary vorticity gradient introduces a retardation proportional to $\beta$ and the solution represents a stable interfacial wave travelling more slowly than the mean-flow. Consequently, in the frame of the spectral model, where $U_{s}=0$, the waves in a $\beta$-plane model would appear to be travelling "westward".

When $\beta \approx \mathcal{O}\left(\mathcal{F}^{\frac{1}{2}}\right)$, so that the system is weakly dispersive, the multiple-scales approximation to the two-layer equations was found to be of the form

$\dot{A}=C A-A V$,

$V=|A|^{2}$,

where $C$ indicates a complex-valued coefficient. Following Moroz (1981), the details of the coefficients have been omitted.

Equations (4) are similar to those obtained for the strongly dissipative system on an $f$-plane (see $\mathrm{I}$ ), except for the presence of a complex coefficient $C$ in the equations. This changes the initial bifurcation from the trivial solution $A=0$ from a pitchfork to a Hopf bifurcation. As in I, the amplitude of the final wave solution is independent of the initial state of the system.

\subsection{Weakly dissipative systems: $r=O\left(\mathcal{F}^{\frac{1}{2}}\right)$}

Brindley and Moroz (1980) showed that the inclusion of a weak $\beta$-effect in the multiple-scales analysis for a weakly dissipative system also leads to a complexification of the coefficients so that the equations governing the weakly dissipative, weakly dispersive regime become:

$$
\ddot{A}=C A-C \dot{A}-A V,
$$$$
\dot{V}+V=|\dot{A}|^{2}+|A|^{2}
$$

As above, the trivial solution is always a fixed point of this system, losing stability in a Hopf bifurcation as the Froude number, $F$, is increased through a critical value, $F_{c}$, given by

$U_{d}^{2}=\frac{1}{\left(2 F_{c}-K^{2}\right)}\left[\frac{F_{c}^{2} \beta^{2}}{K^{2}\left(K^{2}+F_{c}\right)^{2}}+\frac{K^{2} r^{2}}{k_{n}^{2}}\right]$.
Equations (5) have been shown by Gibbon and McGuinness (1982) to be equivalent to the complex Lorenz equations:

$$
\begin{aligned}
& \dot{X}=-\sigma X+\sigma Y, \\
& \dot{Y}=R X-a Y-X Z, \\
& \dot{Z}=-b Z+\frac{1}{2}\left(X^{*} Y+X Y^{*}\right),
\end{aligned}
$$

where $\sigma$ and $b$ are real numbers, $R=R_{1}+i R_{2}$ and $a=$ $1-i e$. In contrast to the original Lorenz equations, therefore, the "Rayleigh number", $R$, is now complex. To the best of our knowledge, the complex Lorenz equations have not been as thoroughly investigated as the original Lorenz equations, and very little is known about the bifurcations that occur in the weakly dispersive, weakly dissipative regime. We now summarize what is known.

\subsection{The complex Lorenz equations}

The first comprehensive study of Eqs. (7) was performed by Fowler et al. (1982), who showed that as $R_{1}$ is increased, the trivial solution undergoes a supercritical Hopf bifurcation to a stable travelling wave. If $R_{1}$ is increased further, the travelling wave loses stability to a travelling wave with a periodically modulated amplitude. After performing a number of initial value numerical integrations, Fowler et al. (1982) concluded that the transition to this amplitude vacillation was via a subcritical Hopf bifurcation to an invariant torus and that frequency-locking between the drift frequency of the travelling wave and the frequency of the amplitude vacillation was not possible. They observed that for $R_{1}=40$ and $R_{2} \rightarrow 0$, the torus underwent a sequence of period-doubling bifurcations and remarked that "the effect of complexification is to convert oscillatory states into "doubly" oscillatory ones ... and there is no particular reason to suppose the rich behavior of the real Lorenz equations is otherwise modified", although no explanation was offered as to how the "extra oscillatory motion" arose.

The nature of the torus in the complex Lorenz equations was investigated in detail by Fowler and McGuinness (1984), who showed that as $R_{2} \rightarrow 0$ and $R_{1} \rightarrow \infty$, the drift frequency associated with the travelling wave tended to a value that was half that of the frequency of the amplitude oscillation. In this way, the torus reduced to a limit cycle at $R_{2}=0$, in agreement with the real Lorenz equations. An averaging technique was then used to suggest that a homoclinic explosion of tori, occurring as $R_{1} \rightarrow \infty$, was the origin of the period-doubling bifurcations observed as $R_{2} \rightarrow 0$.

\subsection{Inviscid systems}

Pedlosky (1970) and Moroz (1981) extended the multiplescales approach to include weakly dispersive, inviscid systems and obtained

$\ddot{A}=C A-C \dot{A}-A V$,

$\dot{V}=|\dot{A}|^{2}$ 
for the evolution of the slowly-varying amplitude. The critical value of the Froude number at which linear instability occurred was given by

$$
U_{d}=\frac{\beta F_{c}}{K^{2}\left(4 F_{c}^{2}-K^{4}\right)^{\frac{1}{2}}},
$$

which differs from the critical value given by Eq. (6) in the limit $r \rightarrow 0$ :

$U_{d}=\frac{\beta F_{c}}{K\left(2 F_{c}-K^{2}\right)^{\frac{1}{2}}\left(K^{2}+F_{c}\right)}$.

Equation (10) shows that the critical shear for instability to occur in the inviscid limit of viscous theory is less than that predicted by purely inviscid theory. This situation has been discussed by Holopainen (1961), Newell (1972), Romea (1977) and Pedlosky (1981b), and suggests that the combined effects of a $\beta$-effect and viscosity can destabilize an otherwise stable shear flow.

\section{Bifurcations in the weakly dispersive regime}

\subsection{The spectral amplitude equations on a $\beta$-plane}

The spectral amplitude equations for baroclinic instability on a $\beta$-plane (cf Eq. (9) of I) take the form:

$$
\begin{aligned}
\dot{X} & =-\left(\Delta_{s}+i \beta_{s}\right) X+\left(1+\frac{\gamma_{s}}{v_{s}} Z\right) Y, \\
\dot{Y} & =-\left(\Delta_{d}+i \beta_{d}\right) Y+v_{s}\left(v_{d}+\gamma_{d} Z\right) X, \\
\dot{Z} & =-\bar{\Delta} Z \pm \frac{\bar{\gamma}}{2 v_{s}}\left(X^{*} Y+X Y^{*}\right),
\end{aligned}
$$

where $(X, Y, Z)=\left(A_{s}+i B_{s},-i v_{s}\left(A_{d}+i B_{d}\right), X_{d}\right)$ (see Lovegrove et al., 2001, hereafter referred to as Part I, for further details), the dot indicates differentiation with respect to time, and the coefficients are given in Appendix A. As in the $f$-plane limit, the trivial solution $(X, Y, Z)=(0,0,0)$ is always a fixed point of Eqs. (11), and its stability can be studied by linearizing the equations about the fixed point and computing the eigenvalues to give

$$
\begin{aligned}
& \lambda_{1,2,3,4}=-\frac{\left(\Delta_{s}+\Delta_{d} \mp i\left(\beta_{s}+\beta_{d}\right)\right)}{2} \\
& \pm \frac{\left[\left(\Delta_{s}-\Delta_{d} \mp i\left(\beta_{s}-\beta_{d}\right)\right)^{2}+4 v_{s} v_{d}\right]^{\frac{1}{2}}}{2}, \\
& \lambda_{5}=-\bar{\Delta} .
\end{aligned}
$$

Since $\bar{\Delta}$ is proportional to the dissipation, the real eigenvalue $\lambda_{5}$ will clearly be negative for all non-zero values of $r$. The remaining eigenvalues form two complex-conjugate pairs and the system loses stability in a Hopf bifurcation when the real part of one of these pairs passes through zero. This occurs when

$$
\frac{v_{s} v_{d}}{\Delta_{s} \Delta_{d}}=1+\frac{\left(\beta_{s}-\beta_{d}\right)^{2}}{\left(\Delta_{s}+\Delta_{d}\right)^{2}},
$$

which is the same as the condition required for instability on an $f$-plane (see Part I), apart from the addition of a modifying term due to the presence of the $\beta$-effect. Substituting from Appendix A for the coefficients in Eq. (13), yields the following condition for a Hopf bifurcation to occur:

$$
\begin{aligned}
U_{d}^{2} & =\frac{1}{\left(2 F_{c}-K^{2}\right)}\left[\frac{F_{c}^{2} \beta^{2}}{K^{2}\left(K^{2}+F_{c}\right)^{2}}\right. \\
& \left.+\frac{K^{2} r^{2}}{k_{n}^{2}}\left(1+r \epsilon K^{2}\right)^{2}\right],
\end{aligned}
$$

where $r$ is a dissipation parameter, $F$ is the Froude number, $\epsilon$ is the Rossby number, $U_{d}$ is the zonal velocity difference between the layers, $K$ is the total wave number and $k_{n}$ is the zonal wave number. In the absence of horizontal momentum diffusion, Eq. (14) recovers the multiple-scales condition for instability in dispersive, dissipative systems given in Eq. (6). The appearance of a Hopf bifurcation in a dispersive regime, in contrast to the pitchfork of revolution that was found to be present in the $f$-plane system, is related to the symmetry differences between the $\beta$-plane and the $f$-plane spectral amplitude equations as we now discuss.

\section{$3.2 S O(2)$ symmetry}

Knobloch (1996) has examined the effect of symmetry changes on certain bifurcations, and his ideas will now be applied to the $\beta$-plane spectral amplitude equations. The $\beta$ effect may be incorporated into a channel model by introducing a slope to the upper and lower boundaries to represent the presence of a planetary vorticity gradient. As a result, the system is no longer symmetric under a reflection in a plane cutting across the channel and there is now a preferred direction in the system. When $\beta \neq 0$, Eq. (11) remain equivariant under the action of the rotation symmetry $s$, but is no longer equivariant under the action of the reflection symmetry $\rho$ (cf Part I), i.e. $f(s \mathrm{x})=s f(\mathrm{x})$, but $f(\rho \mathrm{x}) \neq \rho f(\mathrm{x})$ and, therefore, only has $S O(2)$ symmetry. Knobloch has shown that changing the symmetry of a system from $O$ (2) to $S O$ (2) introduces a new frequency, $\omega$, into the system. In the $f$-plane system, the initial bifurcation from the trivial solution created a stationary wave in which the spatial phase, $\phi$, remained constant with time (see Part I). In the $\beta$-plane system, however, the introduction of a preferred direction and the consequent change of symmetry means that the spatial phase of the wave formed in the initial bifurcation is no longer constant, but instead oscillates with the new frequency, $\omega$. As a result, the $f$-plane continuum of fixed points representing stationary waves is no longer possible; instead we obtain a travelling wave with a drift frequency, $\omega$. This limit cycle is created in a Hopf bifurcation, which replaces the pitchfork of revolution that was present on the $f$-plane.

Knobloch (1996) also showed that any secondary bifurcations present in a system will be affected by the symmetry of that system. In Part I, we showed that there was a subcritical Hopf bifurcation present in the weakly dissipative $f$-plane system. It was argued that this subcritical Hopf bifurcation 
leads to a continuum of unstable limit cycles (i.e. a torus foliated by periodic orbits). Just as breaking the reflection symmetry $\rho$ leads to the creation of a limit cycle from a continuum of fixed points, so it will also lead to the creation of a torus from a continuum of limit cycles. By the symmetry arguments presented here, one can therefore see that the $\beta$ plane Hopf bifurcation must be a bifurcation to an invariant torus (cf Fowler and McGuinness, 1984).

\subsection{Transformation to a co-rotating frame}

In the numerical solution of the $f$-plane system (carried out in Part I, as in the present work, using AUTO86; Doedel, 1981; Doedel and Kernevez, 1986), it was possible to branchswitch at the subcritical Hopf bifurcation in order to trace out the curve of unstable periodic orbits. This led to the detection of the homoclinic connection. To perform a similar analysis for the $\beta$-plane system, it is necessary to branchswitch at the bifurcation to an invariant torus. Unfortunately, AUTO86 does not possess the facility to do this. This leads to two possible methods for investigating the bifurcations on a $\beta$-plane; either a purely analytical or a hybrid approach involving some analytical work combined with solution continuation may be used.

The first approach was employed by Fowler and McGuinness (1984) to deduce that the torus bifurcation was subcritical, but it rapidly became complicated and incapable of investigating any subsequent bifurcations. In the second approach, used by Weng et al. (1986) in their study of the Eady problem, the equations of motion were transformed into a reference frame rotating with an angular frequency $\omega$. The travelling wave became a single fixed point, and the bifurcation to an invariant torus became a Hopf bifurcation. Branchswitching using AUTO86 is now feasible, and it is this approach that we adopt here.

The amplitude of the travelling wave must be calculated analytically in order to provide a point for the AUTO86 continuation algorithm. Following Weng et al. (1986) we introduce

$$
\begin{gathered}
\left(A_{s}, B_{s}\right)=\left(|S| \cos \left(\omega t+\phi_{0}\right),\right. \\
\left.|S| \sin \left(\omega t+\phi_{0}\right)\right), \\
\left(A_{d}, B_{d}\right)=\left(|D| \cos \left(\omega t+\phi_{0}+\Theta\right),\right. \\
\left.|D| \sin \left(\omega t+\phi_{0}+\Theta\right)\right),
\end{gathered}
$$

where $S$ is the amplitude of the barotropic wave; $D$ is the amplitude of the baroclinic wave; $\omega$ is the frequency of the travelling wave; and $\Theta$ is the zonal phase difference between the barotropic and baroclinic waves (which determines the vertical phase tilt of the resultant wave). Due to the presence of the translational symmetry in the weakly dispersive system, there is no preferred initial position for a wave to form, and so an arbitrary initial spatial phase, $\phi_{0}$, must be included for full generality.

It is well-known (e.g. Pedlosky, 1964) that, in order for baroclinic instability to occur, the wave must tilt to the west with height. This allows for the growing disturbance to draw energy from the mean-flow. A negative value of $\Theta$ is consistent with a westward tilt, while a positive value of $\Theta$ indicates an eastward tilt of the disturbance with height.

If we define

$\left(A_{d}, B_{d}\right)=\left(\widehat{P} A_{s}+\widehat{Q} B_{s},-\widehat{Q} A_{s}+\widehat{P} B_{s}\right)$,

it follows from (15a) and (15b) that

$\left(\dot{A_{s}}, \dot{B}_{s}, \dot{A_{d}}, \dot{B}_{d}, \dot{X_{d}}\right)=\left(-\omega B_{s}, \omega A_{s},-\omega B_{d}, \omega A_{d}, 0\right)$,

with

$$
\begin{aligned}
& A_{s} A_{d}+B_{s} B_{d}=\widehat{P}|S|^{2}, \\
& A_{s} B_{d}-B_{s} A_{d}=-\widehat{Q}|S|^{2}, \\
& A_{d}{ }^{2}+B_{d}{ }^{2}=|D|^{2}=\left(\widehat{P}^{2}+\widehat{Q}^{2}\right)|S|^{2} .
\end{aligned}
$$

Substitution of the above into Eqs. (11) and solving for the steady travelling wave, we obtain:

$$
\begin{aligned}
\omega= & -\frac{\left(\beta_{s} \Delta_{d}+\beta_{d} \Delta_{s}\right)}{\Delta_{s}+\Delta_{d}}, \\
|S|^{2}= & \frac{\Delta_{d} \bar{\Delta}}{\gamma_{d} \bar{\gamma}} \Xi-\frac{v_{d} \bar{\Delta}}{2 \gamma_{d}^{2} \bar{\gamma} \Delta_{s}} \tau \\
& +\frac{v_{d} \bar{\Delta}}{2 \gamma_{d}^{2} \bar{\gamma} \Delta_{s}}\left[\tau^{2}-4 \Xi \Delta_{s} \Delta_{d} \gamma_{s} \gamma_{d}\right]^{\frac{1}{2}}, \\
\widehat{Q}= & \frac{v_{d}}{\left[\Delta_{d} \Xi-\frac{\gamma_{d} \bar{\gamma}|S|^{2}}{\bar{\Delta}}\right]}, \\
\widehat{P}= & \widehat{Q} \frac{\left(\omega+\beta_{s}\right)}{\Delta_{s}}, \\
X_{d}= & -\frac{\bar{\gamma}}{\bar{\Delta}} \widehat{Q}|S|^{2},
\end{aligned}
$$

where

$$
\Xi=1+\frac{\left(\beta_{s}-\beta_{d}\right)^{2}}{\Delta_{s}+\Delta_{d}^{2}} \quad \text { and } \quad \tau=v_{s} \gamma_{d}-v_{d} \gamma_{s} .
$$

Hence,

$$
\begin{gathered}
\dot{S}=-\Delta_{s} S-\left(v_{s}+\gamma_{s} X_{d}\right) D \sin \Theta, \\
\dot{D}=-\Delta_{d} S+\left(v_{d}+\gamma_{d} X_{d}\right) D \sin \Theta, \\
\dot{X_{d}}=-\bar{\Delta} X_{d}+\bar{\gamma} S D \sin \Theta, \\
\dot{\Theta}=\beta_{s}-\beta_{d}+\frac{S}{D}\left(v_{d}+\gamma_{d} X_{d}\right) \cos \Theta \\
\quad-\frac{D}{S}\left(v_{s}+\gamma_{s} X_{d}\right) \cos \Theta .
\end{gathered}
$$

Note that in Eqs. (21) both $S$ and $D$ are positive quantities and the modulus signs have been dropped for convenience. Also, these equations are undefined when either $S$ or $D$ is zero. This is in contrast to the original untransformed equations where $S=D=0$ is a valid solution and means that the transformed equations can only be used for a solution continuation once a wave is present in the system. Hence, for any numerical solution continuation using AUTO86, we used the untransformed equations of motion to determine the point at which the trivial solution becomes unstable. Once this has been found, the transformed equations could then be used in all subsequent analyses. 


\section{Bifurcations in a dissipative system}

\subsection{Strong dissipation}

The analysis of the strongly dissipative system was performed for $r=0.2, \epsilon=0.05$ and $\beta=0.1$, with the Froude number as the bifurcation parameter. Calculations show that the trivial solution loses stability to a travelling wave in a Hopf bifurcation at $F=5.49$, which remains stable over the range of Froude numbers considered. After the creation of the travelling wave $\phi$ becomes negative, indicating a westward tilt with height required for the formation of an interfacial wave.

Numerical integrations of the untransformed $\beta$-plane equations of motion clearly show the differences between the strongly dissipative, weakly dispersive system and the strongly dissipative $f$-plane system (see Part I). In both cases, there was a stable wave present in the system, but in the $\beta$-plane case, the spatial phase was time-dependent with a saw-tooth periodic profile, reflecting the travelling nature of the wave, whereas in the $f$-plane, the wave was stationary.

\subsection{Weak dissipation}

Fowler et al. (1982) showed that the weakly dissipative multiple-scales equations are equivalent to the complex Lorenz equations. To see if this also holds for the spectral equations we chose $r=0.02$ and $\beta=0.1$. The untransformed equations were used to locate the initial Hopf bifurcation and then the transformed equations were used to trace out the branch of periodic orbits created in the Hopf bifurcation. The resulting bifurcation diagram for the barotropic wave amplitude, $S$, is shown in Fig. 1.

As in the strongly dissipative, weakly dispersive system, the trivial solution loses stability via a Hopf bifurcation to a travelling wave, which subsequently loses stability at $F=6.17$ in a secondary Hopf bifurcation. Branchswitching at this torus bifurcation showed the bifurcation to be subcritical, as in Fowler and McGuinness (1984). The branch of unstable tori corresponds to travelling wave solutions in which the amplitude varies periodically in time, in the same way that the unstable periodic solutions involved in the subcritical Hopf bifurcation on the $f$-plane represented a stationary wave with a vacillating amplitude (see Part I for further details). As the Froude number was decreased, the minimum amplitude of the barotropic wave decreased towards zero, so that the unstable tori appear to become homoclinic to the fixed point representing the unstable trivial solution. However, no homoclinic connection takes place; instead, the branch of unstable tori becomes stabilized via a saddle-node bifurcation at $F=5.65$. This is in contrast to the homoclinic connection, for the $f$-plane model.

The branch of tori then undergoes a series of period-doubling bifurcations, beginning at $F=5.67$. An enlarged view of the first few of these bifurcations is shown in Fig. 2. At larger values of the Froude number, an inverse period-doubling cas- cade takes place, in which the original branch of torus solutions re-stabilies.

Thus, there are no homoclinic connections present in the weakly dissipative, weakly dispersive spectral amplitude equations, thereby disagreeing with the hypothesis put forward by Fowler and McGuinness (1984) that the complex Lorenz equations possess a homoclinic connection. The causes of this apparent discrepancy will now be examined.

\subsection{Symmetry and homoclinic cycles}

Chossat (1993) recently examined the effect of symmetry breaking on homoclinic cycles. He showed that perturbations which break the reflectional symmetry of an $O$ (2) system generically cause a homoclinic connection to bifurcate to quasi-periodic flow on a 2-torus. The resulting $S O$ (2) action adds one more frequency by allowing for the solution to "drift along the group orbit" and these two frequencies cause the quasi-periodic motion on a torus. In our problem, the "drift along the group orbit" is the frequency $\omega$ of the travelling wave. Applying Chossat's idea to this study shows, therefore, that the absence of a homoclinic connection described above is to be expected from symmetry arguments. This, together with evidence from the numerical solution continuation, also suggests the absence of a homoclinic connection in the weakly dispersive, multiple-scales equations.

A $\beta$-effect, therefore, destroys the Lorenzian homoclinic connection and produces a quasi-periodic motion in its place. This, in turn, suggests that the complex Lorenz equations should be viewed as a perturbed form of the real Lorenz equations and there is no reason a priori to suppose that the Lorenzian behavior of the $f$-plane spectral amplitude equations should persist in the weakly dispersive spectral amplitude equations. Indeed, in Part I, it was shown that the existence of a homoclinic connection in the $f$-plane system was crucial to the creation of chaotic dynamics. This implies that if chaotic motion is present in the weakly dispersive system, as suggested by Fowler et al. (1982), it must be created by a different mechanism. The $f$-plane and $\beta$-plane systems, therefore, possess fundamentally different dynamics and the $f$-plane model should not be regarded simply as the $\beta \rightarrow 0$ limit of the $\beta$-plane model.

\subsection{Numerical integration}

A series of numerical integrations was performed to examine the behavior of the system as the Froude number was increased beyond the critical value at which the subcritical torus bifurcation occurred. When $F=4.0$, the trivial solution was stable, while for $F=5.6$, the travelling wave solution was stable. At $F=5.9$, although the solution continuation suggested that the travelling wave solution should still be stable, the small perturbation to the system did not in fact settle down to a steady value but instead, evolved to aperiodic motion. 

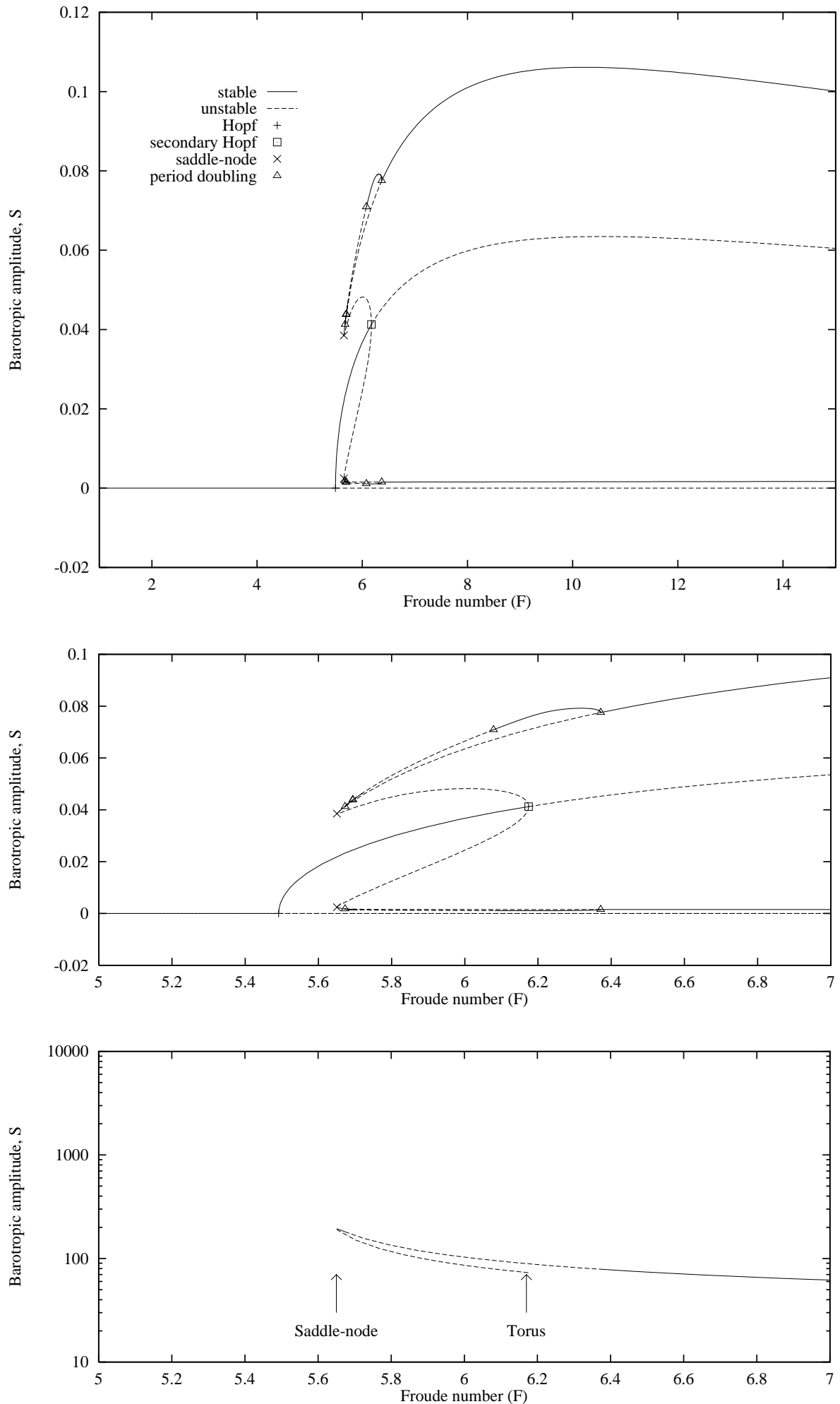

Fig. 1. Bifurcation diagram showing the effect of increasing the Froude number at $r=0.02, \beta=0.1$. The trivial solution lost stability to a travelling wave at $F=5.49$. The stationary wave subsequently became unstable to an unstable torus in a subcritical secondary Hopf (or torus) bifurcation at $F=6.17$. Both the maximum and the minimum amplitudes of this orbit are shown. The branch of unstable tori "flipped back" on itself in a saddle-node bifurcation at $F=5.65$. The stable branch subsequently underwent a period-doubling cascade.

Fig. 2. Enlargement of Fig. 1, showing the first two bifurcations in a perioddoubling cascade. Compare this with Fig. 5 of Part I, which shows the corresponding situation for the $f$-plane.

Fig. 3. Period of the unstable torus born in the subcritical torus bifurcation. Note that although the period increases slightly as the saddle-node bifurcation is approached, but it never approaches infinity.
At higher values of the Froude number, integrations of the transformed equations reproduced the inverse period-doubling cascade reported above. Figure 4 shows the final two inverse period-doubling bifurcations in the sequence. In the transformed, co-rotating frame, the solution at $F=6.5$ appears to be singly-periodic, whereas in the original untransformed frame, the $F=6.5$ solution appears to be quasi- periodic, corresponding to a travelling wave undergoing an amplitude vacillation.

The barotropic wave amplitude, $S$, and the vertical phase, $\Theta$, of the singly-periodic orbit at $F=6.5$ are shown as functions of time in Fig. 5. It can be seen that when $\Theta$ is negative, indicating a westward tilt in the instability, the amplitude of the wave is growing and vice versa. This is in agreement with 


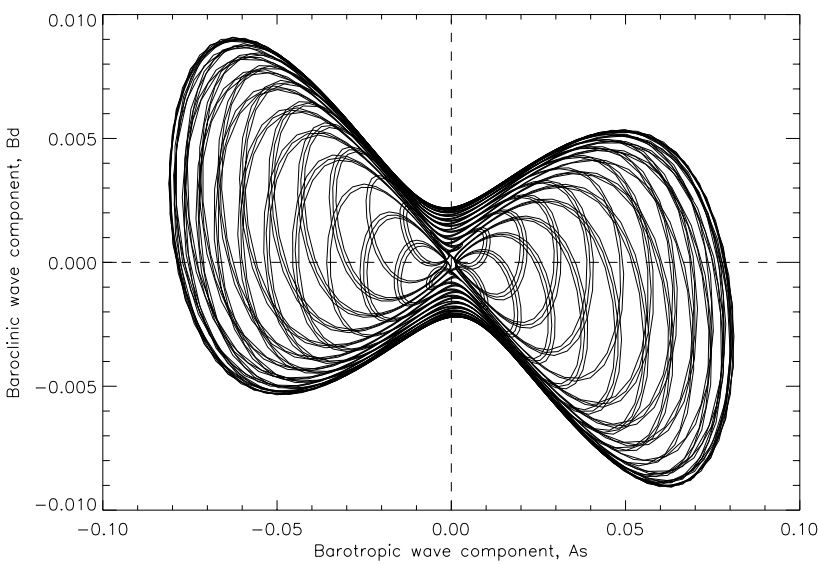

(a) $F=6.5$

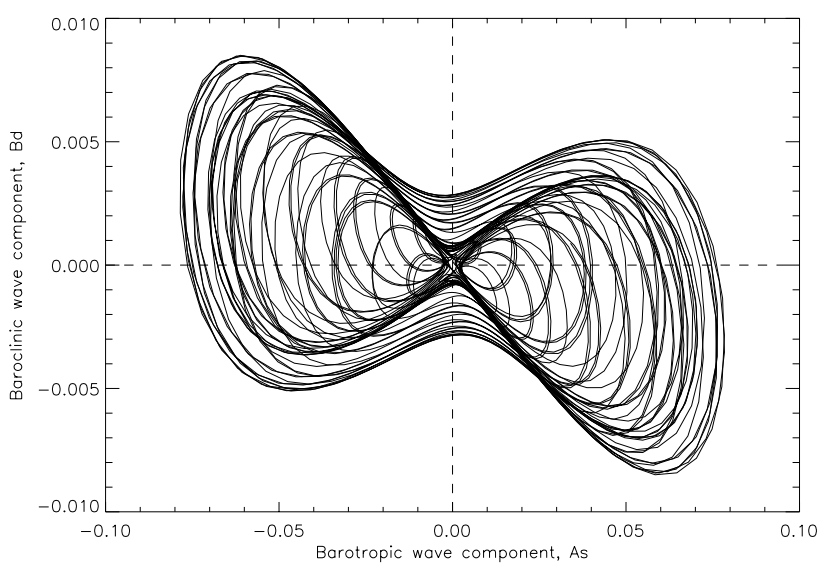

(c) $F=6.26$

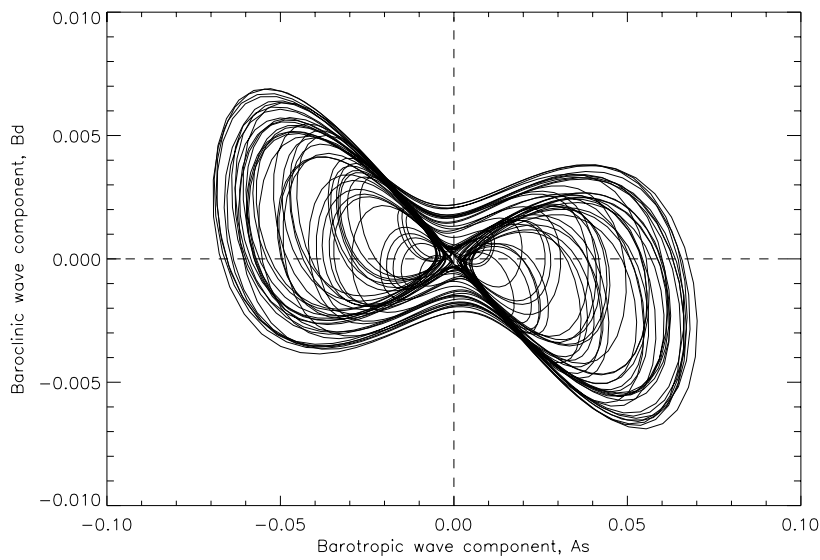

(e) $F=6.05$

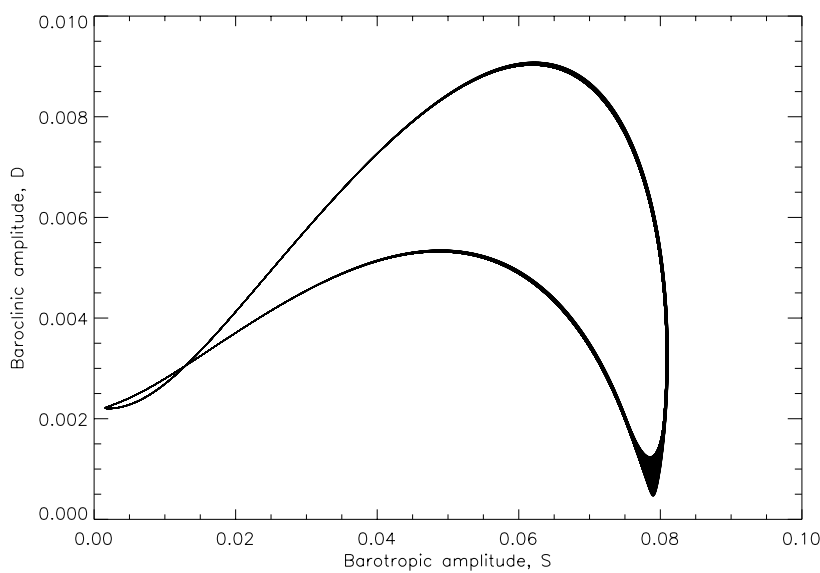

(b) $F=6.5$ (co-rotating frame)

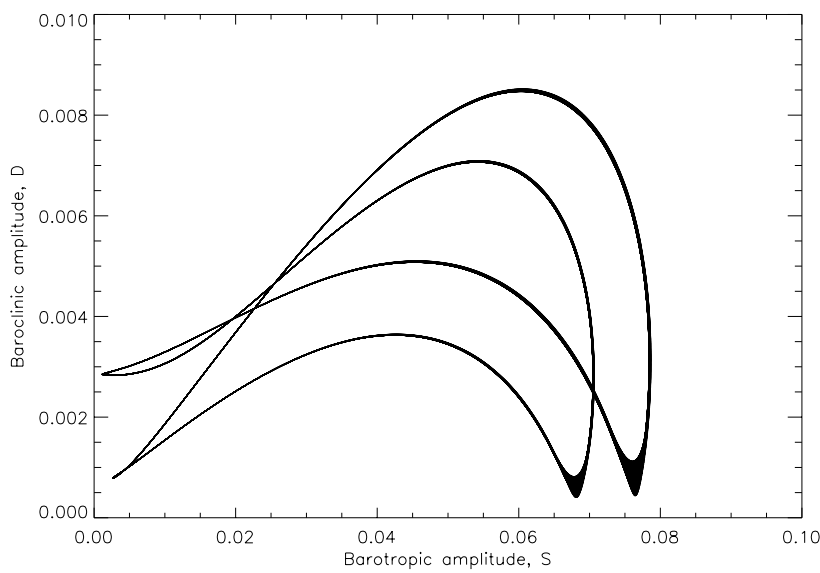

(d) $F=6.26$ (co-rotating frame)

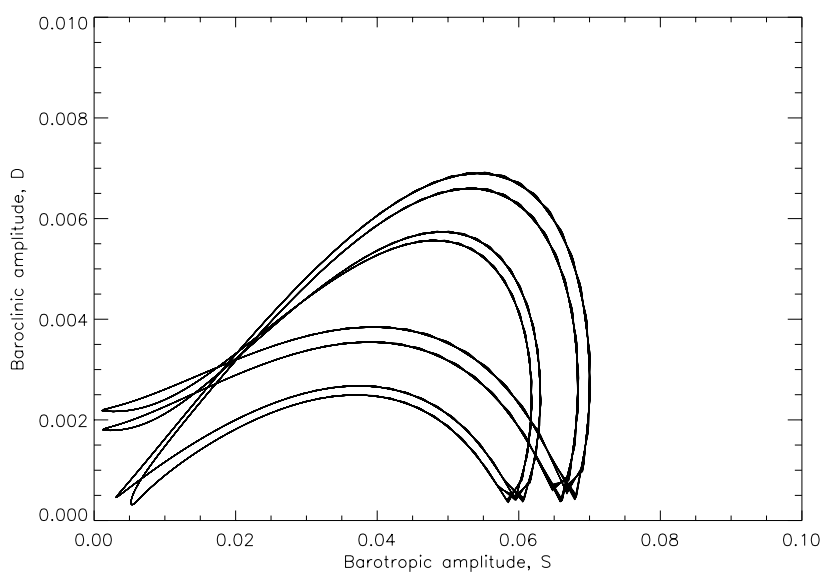

(f) $F=6.05$ (co-rotating frame)

Fig. 4. A series of numerical integrations performed at $r=0.02, \beta=0.1$, showing the last two period-doubling bifurcations in the $\beta$ plane system in both the original, untransformed frame $[(\mathbf{a}),(\mathbf{c})$ and (e)] and in the transformed, co-rotating frame [(b), (d) and (f)]. The period-doubling bifurcations, which occur on a quasi-periodic torus, are only readily observable in the co-rotating frame. Note the apparent widening of the trajectory, in the transformed frame, when the trajectory approaches the $D=0$ axis. This is an artifact of the numerical integration and is due to the finite step-size used. 


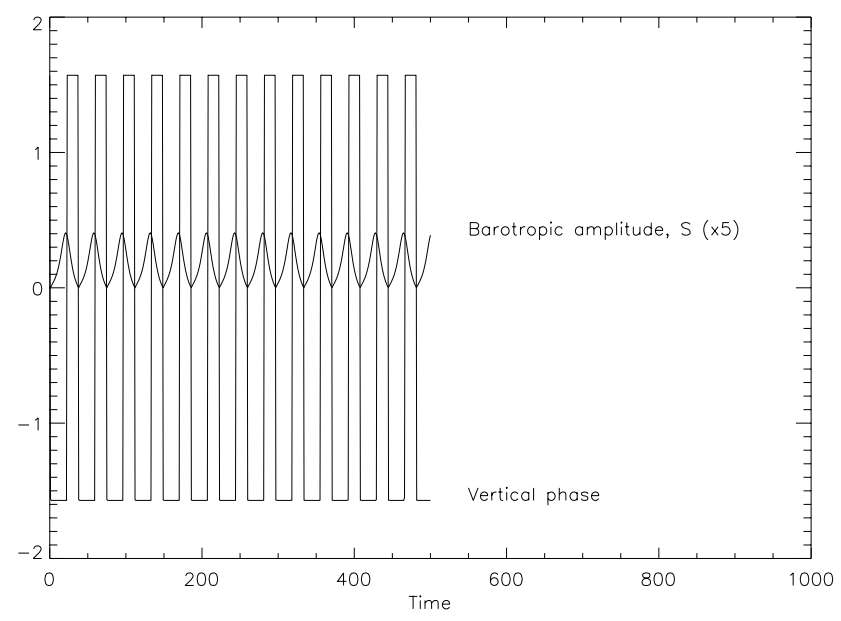

Fig. 5. The vertical phase, $\Theta$, and the barotropic amplitude, $S$, as functions of time, during an amplitude vacillation.

the analysis of Pedlosky (1964), who showed that a westward tilt is necessary for a growing disturbance to extract energy from the mean-flow.

\subsection{Symmetry-breaking}

As $F$ is decreased, the limit cycle in the $f$-plane model undergoes a symmetry-breaking bifurcation before perioddoubling occurs (see Figs. 6a and c). If the bifurcations in the $f$-plane model are re-plotted in the $\left[\left|A_{s}\right|,\left|B_{d}\right|\right]$ plane, as shown in Figs. 6b and d, the symmetry-breaking bifurcation resembles a period-doubling. In the $\beta$-plane model as viewed in the co-rotating frame, Figs. $4 \mathrm{~b}$ and $\mathrm{d}$ show that the bifurcation sequence appears identical to that observed on the $f$-plane, even though the corresponding attractor in the untransformed frame (see Figs. 4a, c and e) appears symmetrical. The first apparent period-doubling in Figs. 4b-d, however, is actually a symmetry-breaking bifurcation, but in the untransformed frame the orbit is rotating and quasi-periodic, and traces out a torus. Due to the asymmetry, one side of the torus is thinner and smaller than the other, but the rotation renders the projection of the overall envelope symmetric.

From this observation it appears that the first period-doubling bifurcation in the $\beta$-plane model corresponds to the symmetry-breaking bifurcation in the $f$-plane system. This apparent anomaly occurs because the reflectional symmetry does not apply in this weakly dispersive regime. The transformed equations are by definition unsymmetric and the periodic solution is free to period-double without undergoing any symmetry-breaking bifurcations (Swift and Wiesenfeld, 1984).

\subsection{Lyapunov exponents}

While it might be difficult to prove that the complex Lorenz equations exhibit chaotic solutions, some evidence for chaotic motion could be obtained by calculating the largest Lyapunov exponent of the system.
Lyapunov exponents measure the average rate of separation of two neighboring points in phase space. In general, a system will have a number of different Lyapunov exponents; each measuring the separation rate in a different direction in phase space. For an $n$-dimensional dynamical system, the Lyapunov exponent is defined by monitoring the long-term evolution of an infinitesimal $n$-sphere of initial conditions. The $i$ th Lyapunov exponent, $h_{i}$, is then

$h_{i}=\lim _{t \rightarrow \infty} \frac{1}{t} \log _{2} \frac{p_{i}(t)}{p_{i}(0)}$,

where $t$ denotes time, and $p_{i}(t)$ is the length of the $i$ th ellipsoidal principal axis. A positive Lyapunov exponent, therefore, indicates expansion, whereas a negative exponent denotes contraction. A system is defined to be chaotic when one of its Lyapunov exponents is positive (e.g. Wolf et al., 1985). In general, Lyapunov exponents must be computed numerically and for the study of the weakly dispersive system, the method of Wolf et al. (1985) for numerically estimating the value of the largest Lyapunov exponent was used. Using this method, an initial value numerical integration, from which the transients have been allowed to decay, is performed. The phase space attractor of the system is then reconstructed using standard techniques, such as those described in Lovegrove (1998). Two neighboring points on this attractor are then tracked for a predetermined length of time, during which the average rate of expansion is calculated. When these two points move "too far apart", two new neighboring points are selected and the process is repeated until the estimates of the Lyapunov exponent converge.

Recall from the two-parameter continuation of bifurcations in the $f$-plane system that, in the $[r, F]$-plane, a solution continuation at a constant value of $F$, using $r$ as a control parameter, should reveal the same principal bifurcation sequence as that observed in solution continuations performed by varying $F$ at constant $r$ (i.e. pitchfork $\rightarrow$ Hopf $\rightarrow$ homoclinic). The same should be true of the weakly dispersive regime. To support this, Fig. 7 shows a solution continuation of the weakly dispersive spectral amplitude equations performed at $F=11$, with $\beta=0.1$ and $\epsilon=0.05$. It can be seen that the bifurcation sequence is indeed the same as was observed in the solution continuation in which $F$ was chosen as the dependent parameter (Fig. 1).

This approach of keeping $F$ constant and varying $r$ for successive integrations was used to calculate the largest Lyapunov exponent of the transformed equations. The Froude number was fixed at $F=11$, and $r$ was increased from $r=0.01$ to $r=0.105$. The result is shown in Fig. 8 in which the largest Lyapunov exponent, together with lines representing three standard deviations on the calculation, is plotted as a function of $r$.

For $r>0.105$, the largest Lyapunov exponent was less than zero. This was consistent with having a stable fixed point representing the travelling wave. As $r$ was decreased below $r=0.105$, the largest Lyapunov exponent became positive and the dynamics became chaotic. Finally, as $r$ 


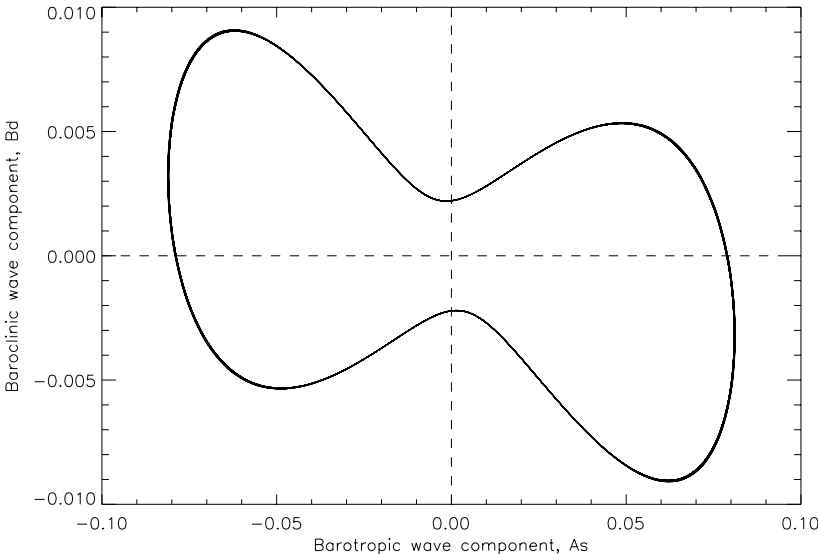

(a) $F=6.5$

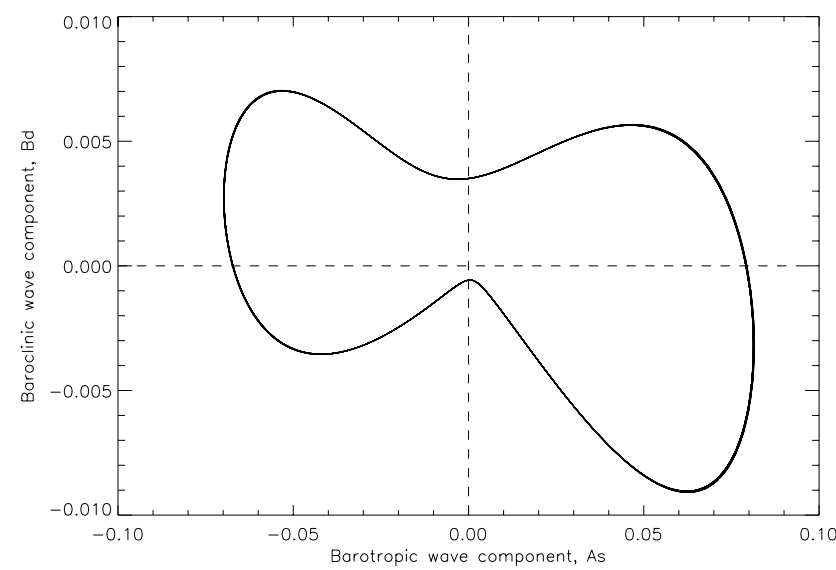

(c) $F=6.26$

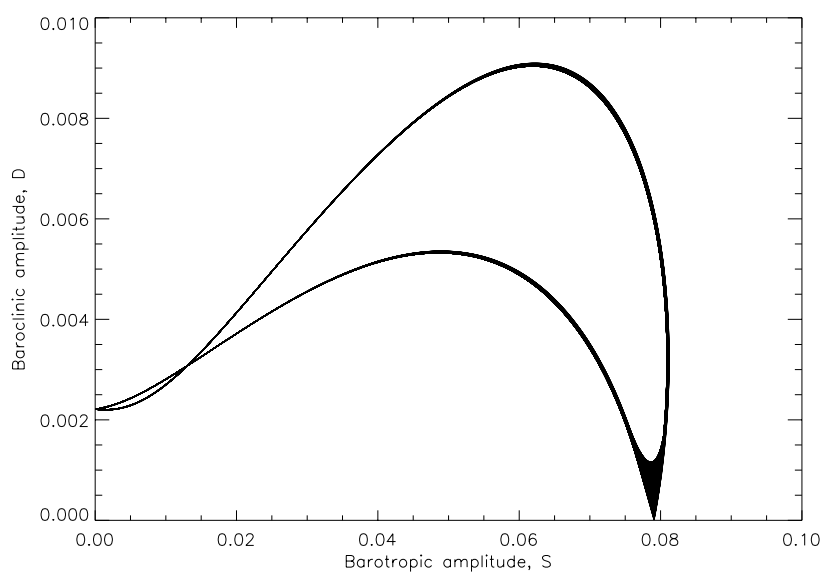

(b) $F=6.5$ (co-rotating frame)

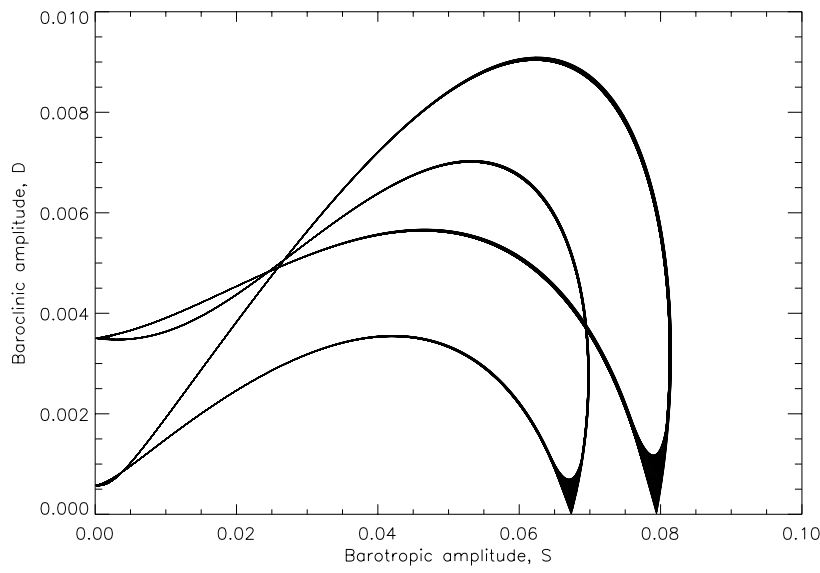

(d) $F=6.26$ (co-rotating frame)

Fig. 6. Integrations performed at $\beta=0$, showing the symmetric and asymmetric $f$-plane limit cycles viewed in the original untransformed frame, and in the co-rotating frame. Note that in the co-rotating frame, the symmetry-breaking bifurcation appears as a period-doubling bifurcation.

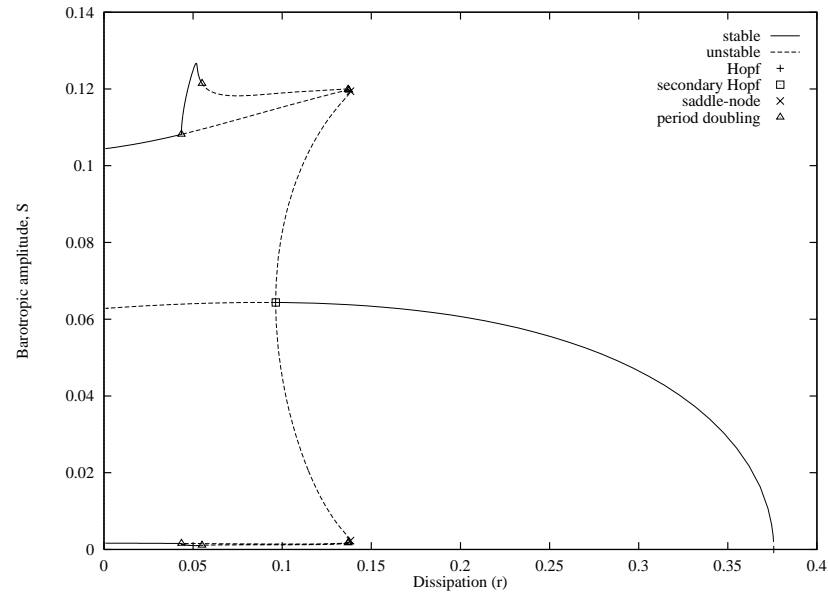

Fig. 7. Bifurcation diagram showing the effect of decreasing dissipation at $\beta=0.1, F=11.0$. Note that the sequence of bifurcations is the same as that encountered in Fig. 1.

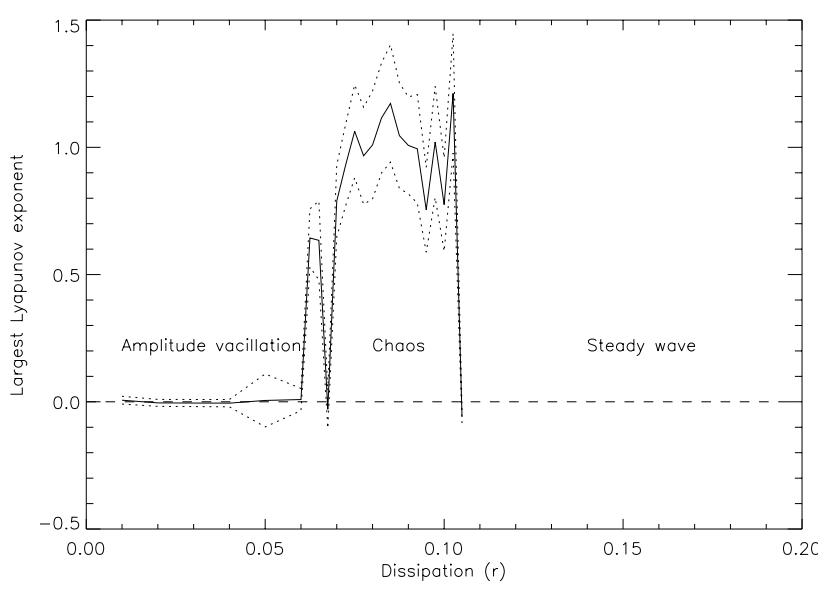

Fig. 8. The largest Lyapunov exponent as a function of the dissipation parameter $r$, at $\beta=0.1, F=11.0$. 
Barotropic amplitude, $\mathrm{S}$

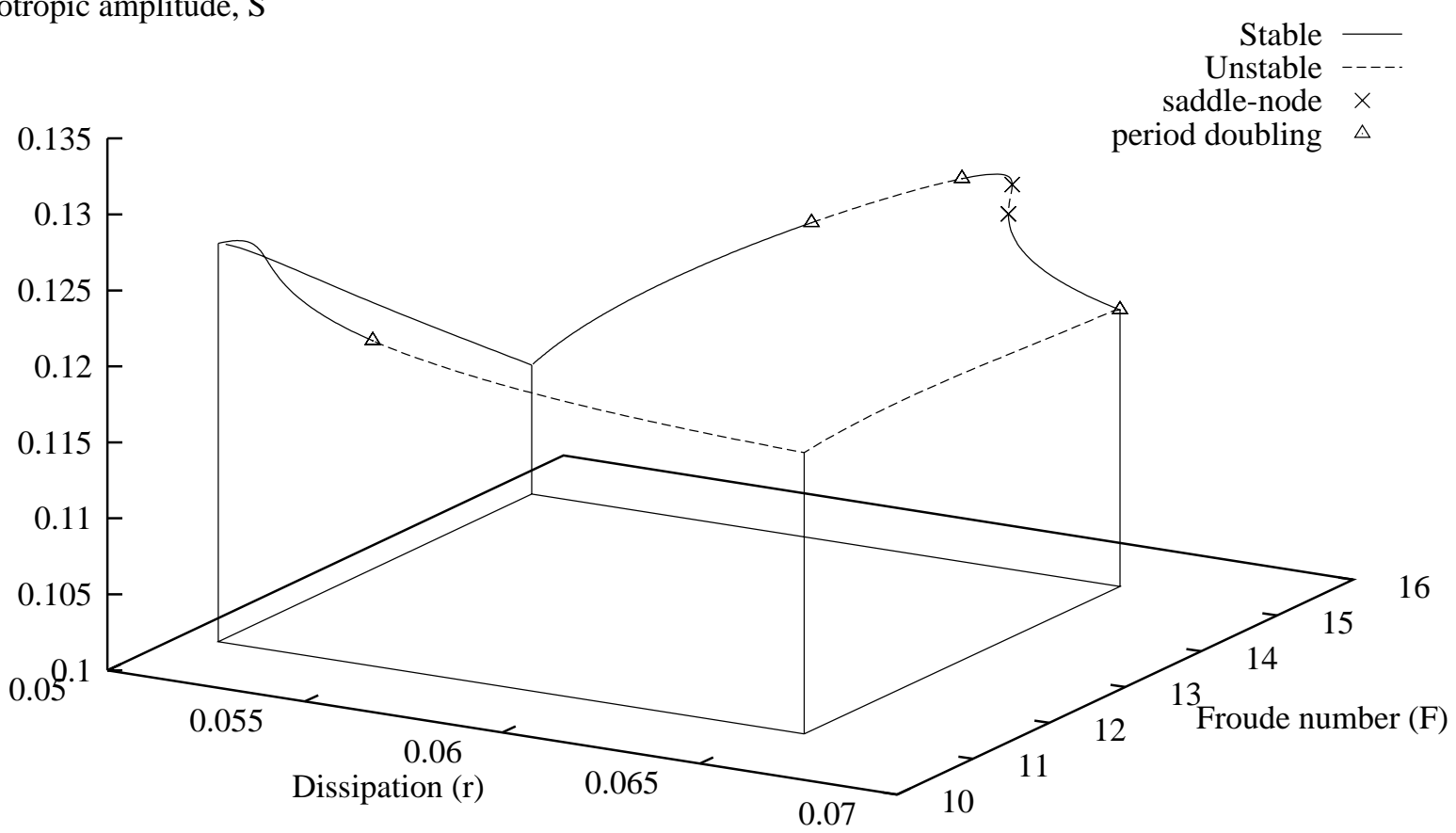

Fig. 9. A series of one-parameter continuations performed around a closed curve in parameter space, enclosing the region surrounding the suspected cusp bifurcation. Since there are no discontinuities in the curve, a cusp bifurcation is present.

is decreased below $r=0.06$, the largest Lyapunov exponent decreased to zero, indicating the presence of the limit cycle, corresponding to the amplitude vacillation regime in this co-rotating reference frame. As the solution continuation has shown that the homoclinic connection present in the $f$-plane system does not persist when $\beta$ becomes non-zero, any chaotic attractor that may be present in the weakly dispersive system must be created by a different mechanism.

\subsection{Cusp bifurcations}

In Fig. 7, the solution branch corresponding to a perioddoubled torus exhibits an unusually large response at $r=0.052$. This appears to be a form of resonant response to the forcing provided by the presence of a $\beta$-effect. As the Froude number is increased to $F=30$, the amplitude response grows larger and the branch "tilts over", creating two new saddle-node bifurcations at $r=0.0737$ and $r=0.0782$ and introducing hysteresis into the system. In addition to the two saddle-node bifurcations, two new period-doubling bifurcations are also created.

Such amplitude responses are commonly observed in forced nonlinear oscillators, e.g. in the forced Duffing equation (Drazin, 1992), and represent a nonlinearly limited resonant response to an external forcing. This phenomena has not been mentioned in previous descriptions of the weakly dispersive regime (e.g. Fowler et al., 1982; Gibbon and McGuinness, 1982; Fowler et al., 1983; Fowler and McGuinness, 1984) and the physical mechanism behind the forcing remains unclear.
Using AUTO86, we performed a two-parameter continuation of the two new saddle-node bifurcations. The loci of the saddle-node bifurcations trace out a "tongue" in the $(r, F)$-plane, converging as the Froude number is decreased from $F=30$ and appearing to meet in a degenerate bifurcation. Unfortunately this could not be shown explicitly using AUTO86, as the two-parameter continuation became inaccurate close to this degenerate bifurcation.

One possibility is for the degenerate bifurcation to be a cusp point. This was confirmed by performing a number of one-parameter continuations of the period-doubled solution branch along the boundary of a region enclosing the suspected cusp bifurcation. The beginning and the end of this path were located at the same point in the $(r, F)$-plane. Since there were no discontinuities in the period-doubled solution branch, the solution at the end of the path could be identified with the solution at the beginning of the path and a cusp bifurcation was, therefore, present. The result of this series of continuations is shown in Fig. 9.

The presence of a cusp bifurcation explains the appearance of the saddle-node bifurcations as the Froude number is increased. It does not, however, seem to provide a mechanism for generating the observed new period-doubling bifurcations. One possible explanation for their appearance is that the strong amplitude response of the system causes the original curve of period-doubled tori to make a close approach to the unstable fixed point at the origin (i.e. $S$ decreases to $S=0.001$ or $1 \%$ of its maximum amplitude). This tendency towards homoclinicity means that the stable and unstable manifolds of the trivial fixed point will closely 


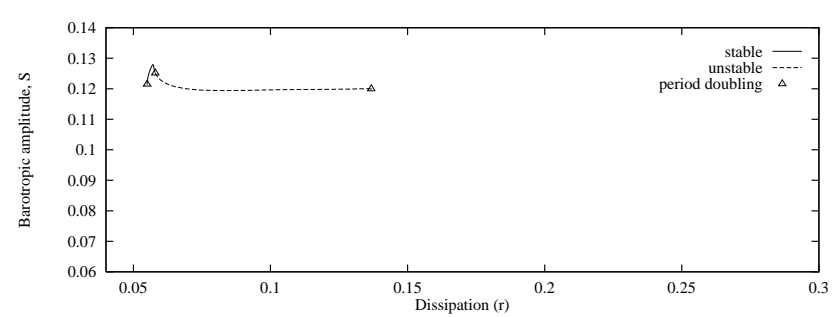

Fig. 10. The branch of period-quadrupled tori arising from the period-doubling bifurcations observed at $F=11.0$.

approach each other. It has been shown that the close approach of stable and unstable manifolds can generate cascades of period-doubling bifurcations (see Guckenheimer and Holmes, 1983).

It was also possible to trace out the branches of solutions emanating from the new period-doubling bifurcations. These solution branches represent curves of period-quadrupled tori. One of these period-quadrupled branches is shown in Fig. 10 for $F=11.0$ and is a smaller facsimile of the original curve of period-doubled tori that was shown in Fig. 9. It has the same resonant response and additional period-doubling bifurcations. One might then reasonably conclude that there are additional cusp points and approaches to homoclinicity to explain the resonant response and period-doubling bifurcations appearing on the branch of period-quadrupled tori.

Further branch switching is possible at the perioddoubling bifurcations on the branch of period-quadrupled tori. These and other continuations show that the structure of the basic branch of period-doubled tori is repeated on ever smaller scales. The "tongue" of saddle-node bifurcations and the presence of cusp bifurcations is reminiscent of the resonant tongues observed in the nonlinear circle map (e.g. Wiggins, 1990).

\section{The circle map}

Arnol'd (1965) introduced the circle map as a method of studying nonlinear coupling between two periodic motions of frequency $\Omega_{j}$ for $j=1,2$ :

$\theta_{n+1}=\left(\theta_{n}+w+k \sin \theta_{n}\right) \bmod (2 \pi)$,

where $\theta_{n}=\theta_{1}\left(t_{n}\right) \bmod (2 \pi)$ and $t_{n}$ is the time at the $n$th piercing of the section $\theta_{2} \bmod (2 \pi)=$ constant; $\theta_{1}$ and $\theta_{2}$ are the two angles describing the torus; and $w=2 \pi \Omega_{1} / \Omega_{2}$.

Defining the rotation number to be $\mathcal{R}=p / q$, Arnol'd found that by varying $w$ and $k$ (with $0 \leq k \leq 1$ ), both periodic orbits with rational values of $\mathcal{R}$ and quasi-periodic orbits with irrational values of $\mathcal{R}$ were possible. Periodic orbits exist in regions known as Arnol'd tongues which come to dominate as the nonlinearity, $k$, is increased. For any given tongue, $\mathcal{R}$ is constant. In between these periodic tongues, quasi-periodic motion exists. The boundaries between the quasi-periodic motion and the frequency-locked peri-

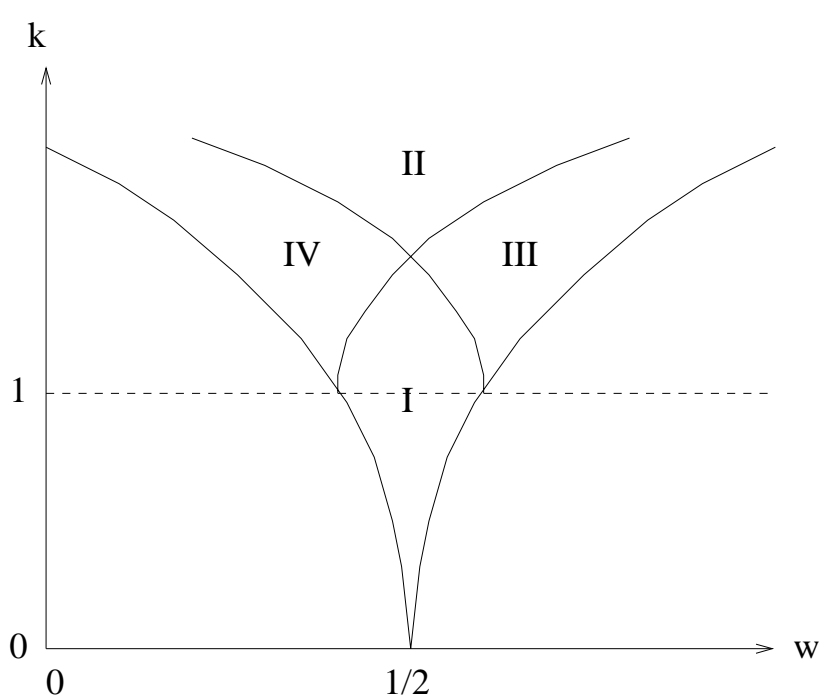

Fig. 11. As $k$ increases, different Arnol'd tongues may overlap. The different regions (I, II, III, and IV) contain qualitatively different dynamics.

odic tongues are defined by lines of saddle-node bifurcations.

The situation for $k>1$ is more complicated and was investigated by Mackay and Tressier (1986) (see also Marek and Schreiber, 1991). In this case, the Arnol'd tongues continue to exist, but now two or more tongues may overlap. When this happens, $\mathcal{R}$ is no longer necessarily a constant for a given tongue, but instead can exist within a rotation interval $R_{\text {int }}=\left[\mathcal{R}^{-}, \mathcal{R}^{+}\right]$. Given a point $(w, k)$ in an Arnol'd tongue, there are four possibilities for the rotation number, $\mathcal{R}$. These four possibilities exist in the four regions shown in Fig. 11:

- In region I, $R_{\text {int }}$ is degenerate, so $\mathcal{R}=\mathcal{R}^{-}=\mathcal{R}^{+}$and the orbit is periodic;

- In region II, $\mathcal{R}$ lies within $R_{\text {int }}$, so the orbit may now either be quasi-periodic and diffuse either clockwise or anticlockwise, or remain periodic;

- In region III, $\mathcal{R}=\mathcal{R}^{-}$and the orbit is quasi-periodic and drifts clockwise;

- In region IV, $\mathcal{R}=\mathcal{R}^{+}$, so the orbit is quasi-periodic and drifts anticlockwise.

\subsection{A circle map in the weakly dispersive regime}

The $\dot{\Theta}$ equation in the transformed set of Eqs. (21) can be re-written as

$\dot{\Theta}=V+\delta(t, \Theta) \cos \Theta$,

where

$$
\begin{aligned}
& V=\beta_{s}-\beta_{d}, \\
& \quad \delta(t, \Theta)=\frac{S}{D}\left(v_{d}+\gamma_{d} X_{d}\right)-\frac{D}{S}\left(v_{s}+\gamma_{s} X_{d}\right) .
\end{aligned}
$$




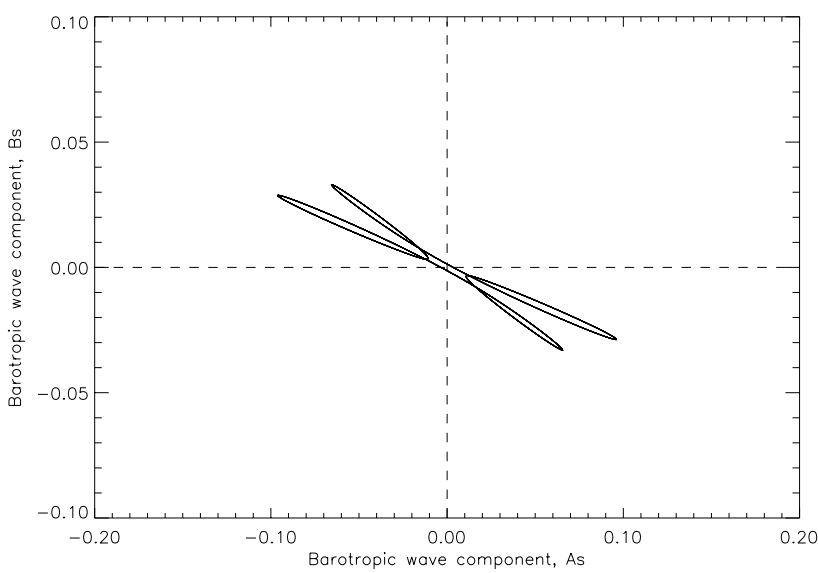

(a) $\left(A_{s}, B_{s}\right)$-plane

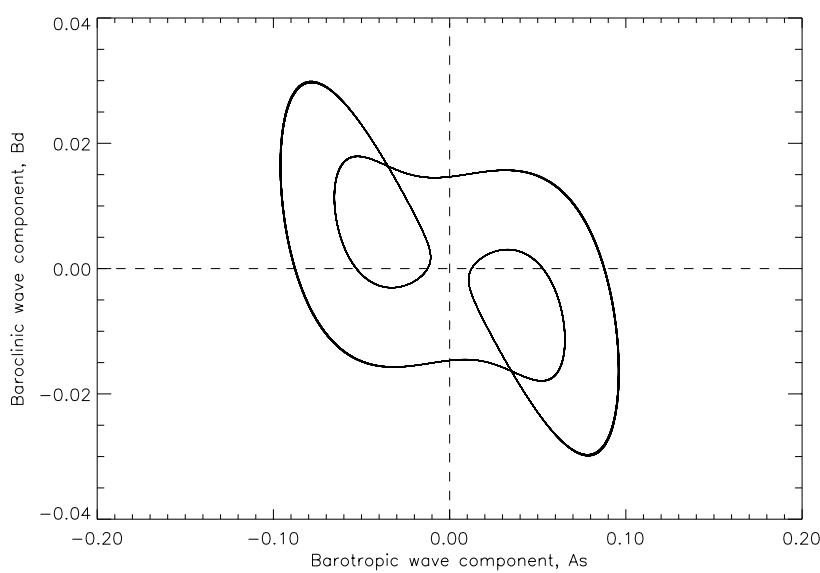

(b) $\left(A_{s}, B_{d}\right)$-plane

Fig. 12. A resonant orbit observed at $r=0.0745, \beta=0.1$ and $R F=30.0$. From the projection onto the $\left[A_{s}, B_{s}\right]$-plane, it can be seen that two vacillation cycles are completed in the time taken for a cycle through the spatial phase. The $\beta$-plane drift frequency, $\omega$, and the vacillation frequency are, therefore, in 1:2 resonance.

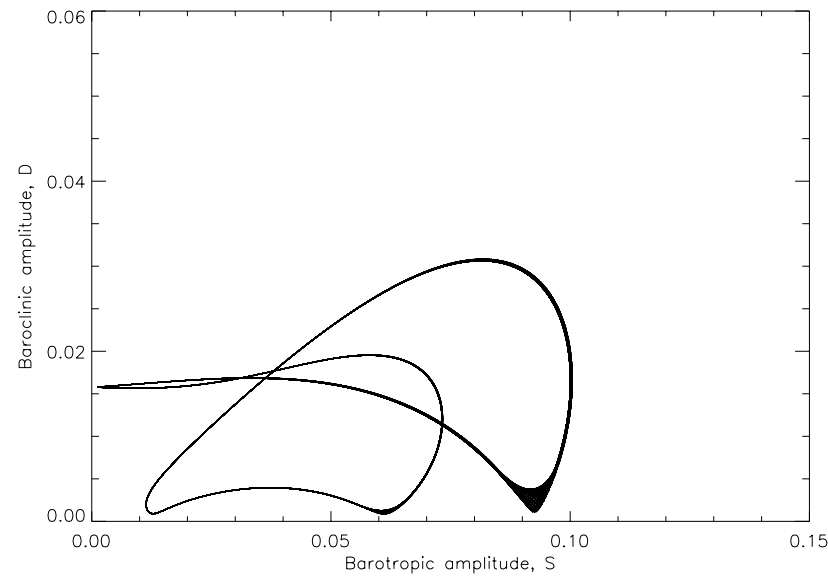

Fig. 13. The resonant orbit at $r=0.0745$ and $F=30.0$, viewed in the transformed co-rotating frame, appears to be a period-two cycle.

Apart from the nonlinear term, Eq. (24) is similar to the circle map given in Eq. (23). This can be readily seen by integrating the linear part of Eq. (24) with respect to time to obtain

$\Theta(t)=\Theta\left(t^{\prime}\right)+V\left(t-t^{\prime}\right)$.

Implicit in Eqs. (21) is a drift frequency, $\omega$, defined by Eq. (19). This can be used to define a surface of section on which to construct the circle map as follows. Let

$t^{\prime}=t_{n}=\frac{2 \pi n}{\omega} \quad$ and $\quad t=t_{n+1}$.

Then $\Theta\left(t^{\prime}\right)=\Theta_{n}$ and $\Theta(t)=\Theta_{n+1}$ so that

$\Theta_{n+1}=\Theta_{n}+w$,

where $w=2 \pi V / \omega$.
While Eq. (24) has a more complicated parametric dependence than in Arnol'd's circle map, nevertheless one might expect to observe phenomena such as frequency-locking in tongues, similar to the Arnol'd tongues in the original circle map, as well as boundaries of these tongues being defined by lines of saddle-node bifurcations.

If frequency-locking does occur in the weakly dissipative, weakly dispersive spectral amplitude equations, then the most likely place to observe it would be inside the regions defined by the saddle-node bifurcations generated by the cusp bifurcation. A number of initial value integrations of the original untransformed equations (see Eq. (9) of Part I) were, therefore, performed in the region $7.37<r<7.81$ at $F=30$.

We found no evidence of frequency locking for most of the interval $0.0737<r<0.0782$ at $F=30$, but when $r=0.0745$ frequency-locking was observed. This solution is shown in Fig. 12, plotted in both the $\left[A_{s}, B_{s}\right]$ and $\left[A_{s}, B_{d}\right]$ planes. There is clearly a rational relation between the drift frequency of the travelling wave and the period-doubled frequency of the amplitude vacillation. Note that this is in contrast to the assertion of Fowler and McGuinness (1984) that there was no frequency locking in the weakly dissipative, weakly dispersive regime.

Figure 13 shows the same frequency-locked orbit in the transformed, co-rotating frame. In this frame the orbit appears to be a period-two orbit. The presence of both frequency-locked and near resonant orbits within the tongue defined by the continuations of the saddle-node bifurcations is reminiscent of the behavior observed in region II of the circle map, where the rotation number is not constant, but exists in an interval $\mathcal{R}_{\text {int }}$, which allows for the orbit to drift clockwise, anticlockwise or remain locked.

Frequency-locked orbits appear to exist only in very small regions of parameter space and, in practice, finding them is 


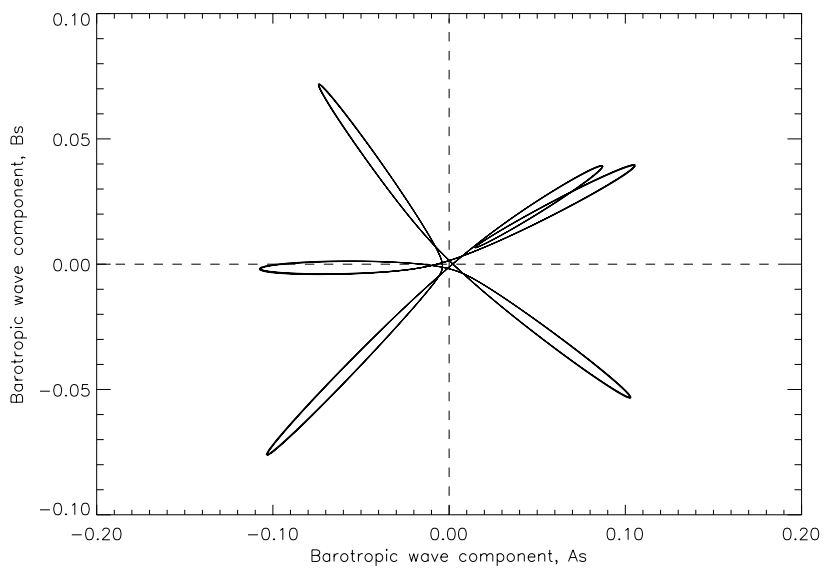

(a) $\left(A_{s}, B_{S}\right)$-plane

Fig. 14. A second resonant orbit, observed at $r=0.060, F=11.0$.

a matter of trial and error. A second frequency-locked orbit, shown in Fig. 14, was found at $R=0.060, \beta=0.1$ and $F=11.0$. This was in a region where, according to the solution continuation shown in Fig. 7, the original branch of period-doubled tori had become unstable. One must conclude, therefore, that this second orbit exists on another branch of solutions.

Figure 15 shows the same resonant orbit viewed in the co-rotating frame. It appears to be a period-six orbit and, therefore, cannot arise from a simple period-doubling of the underlying torus. Therefore, there must be other solution branches in existence that are disconnected and cannot be reached by using AUTO86 to follow solution branches bifurcating from the original branch of torus solutions. Recall that in the co-rotating frame, a symmetry-breaking bifurcation can resemble a period-doubling bifurcation. Therefore, it is possible that the period six orbit in Fig. 14 is actually a period three orbit that has undergone a symmetry-breaking bifurcation.

Sarkovskii (1964) showed that the presence of an orbit of period $p$, with $p$ occurring before another integer $l$ implies the existence of an orbit of period $l$. Li and Yorke (1975) showed that the existence of a period three orbit also implies the existence of an uncountable number of aperiodic orbits. Although Sarkovskii's theorem as originally formulated applies only to mappings of the real line onto itself, its results have since been extended to the circle map, as is relevant here (see Block, 1981; Hidalgo, 1992). By these extensions to Sarkovskii's theorem, the existence of the period six orbit shown in Fig. 13, therefore, implies that the weakly dispersive system contains an infinite number of periodic orbits. If the period six orbit is actually an asymmetric period three orbit, then $\mathrm{Li}$ and Yorke's finding that "period three implies chaos" implies that the weakly dispersive system is actually chaotic, as was concluded from studying the largest Lyapunov exponent of the system.

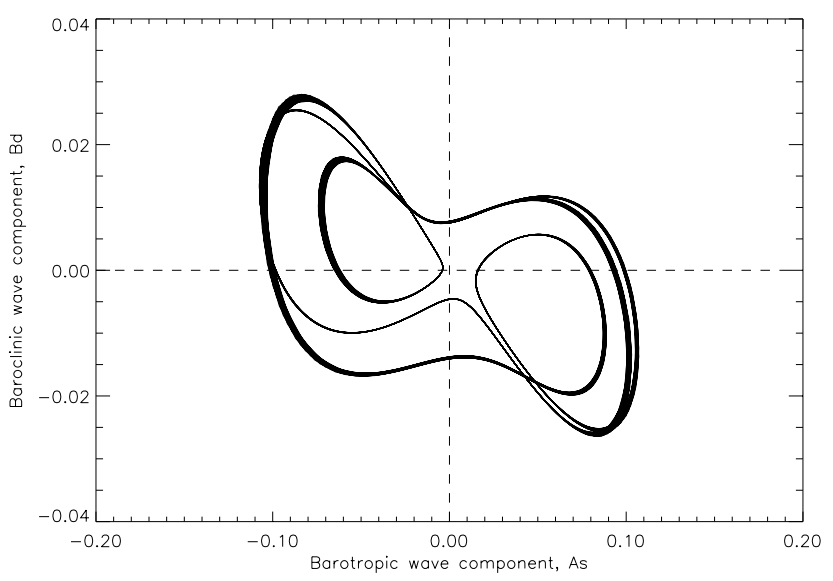

(b) $\left(A_{s}, B_{d}\right)$-plane

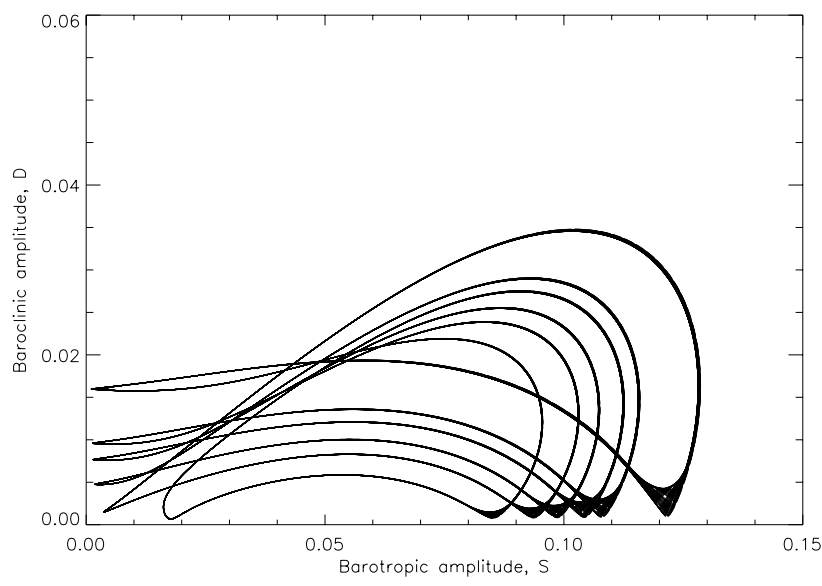

Fig. 15. The second resonant orbit, viewed in the transformed corotating frame, appears to be a period-six orbit.

\section{Bifurcations with increasing $\beta$}

So far we have concentrated on bifurcations occurring for a single value of $\beta$. Pedlosky (1981b) performed a numerical study of the consequences of varying $\beta$ on the chaotic motion observed in the two-layer model. His main conclusion was that such behavior observed on the $f$-plane was strongly suppressed by the addition of a $\beta$-effect. Furthermore, he found that increasing $\beta$ led to a sequence of inverse perioddoubling bifurcations which produced successively less complicated behavior. In this section, we investigate the effects of increasing $\beta$ in the spectral amplitude equations. We shall show that the spectral amplitude equations can reproduce the inverse period-doubling cascade observed by Pedlosky for certain values of $r$. We also show that increasing $\beta$ can lead to other changes in the dynamics of the two-layer model. 


\subsection{Two parameter continuation}

Using AUTO86, a two-parameter continuation of the principal bifurcations observed in the weakly dispersive, weakly dissipative regime was performed in the $(r, \beta)$-plane for $F=$ 11 and $\epsilon=0.05$. Figure 17 shows the curves of Hopf, torus, and saddle-node bifurcations, together with the last perioddoubling bifurcation (which was found to correspond to the symmetry-breaking bifurcation on the $f$-plane).

We see that at higher $\beta$, fewer bifurcations are encountered as $r$ is decreased and consequently, the behavior of the system becomes less complicated. The critical value of $r$ at which the trivial solution loses stability in a Hopf bifurcation decreases until $r=0$ at $\beta \approx 11$. For all higher values of $\beta$, the trivial solution of the two-layer model is stable. We observe also that as $\beta$ is increased, the curves of torus and saddle-node bifurcations converge and appear to merge in a cusp bifurcation at $(r, \beta)=(0.069,1.51)$. The existence of this cusp bifurcation means that as $\beta$ increases through $\beta=1.51$, the torus bifurcation changes from being subcritical to being supercritical and persists to $\beta=1.84$, above which no torus bifurcations are present in the twolayer model.

To clarify this behavior, a series of one parameter continuations using $r$ as the bifurcation parameter was performed at $F=11$ (see Fig. 16). For each run $\beta$ was held constant, but with a different value each time and initialized on the trivial solution at $r=1$. The paths of these continuations in the $(r, \beta)$-plane are also shown in Fig. 17.

It has already been argued that the $\beta$-plane saddle-node bifurcation arises from the $f$-plane homoclinic connection as the $O(2)$ symmetry is broken. For small values of $\beta$, the saddle-node bifurcation and the period-doubling bifurcation are very close together, suggesting that the presence of the period-doubling bifurcation might also be a consequence of breaking the $O(2)$ symmetry on the $f$-plane homoclinic orbit. Following the locus of the period-doubling bifurcation in the $[r, \beta]$ plane reveals that it "bends around" and reappears on the $f$-plane at $r=0.043$. Any chaotic attractor that may be present in the $\beta$-plane system will most likely be created by Feigenbaum's route of an accumulation of perioddoubling bifurcations. An outer bound to the region of parameter space in which chaos can occur is, therefore, provided by the locus of the last period-doubling bifurcation.

A second series of continuations (shown in Fig. 18) was also performed at $F=11$, with $\beta$ chosen as the control parameter and with $r$ held constant for the duration of each run, but varied between continuations. The paths of these continuations on the $(r, \beta)$-plane are also shown in Fig. 17.

From Fig. 18a, it can be seen that decreasing $\beta$ from $\beta=12$ causes the trivial solution to lose stability to a travelling wave in a supercritical Hopf bifurcation. This travelling wave then undergoes a supercritical torus bifurcation to an amplitude vacillation that remains stable for all subsequent values of $\beta$. From Fig. 17, it can be seen that decreasing $\beta$ will only give rise to chaotic motion for $0.069<r<0.14$, since the trivial wave bifurcates to a travelling wave then to an amplitude vacillation in a subcritical torus bifurcation and finally undergoes a series of period-doublings. A oneparameter continuation performed in this region is shown in Fig. 18b.

For $0.14<r<0.38$, the situation was slightly more complicated (see Fig. 18c). The initially stable trivial solution bifurcated to a travelling wave as $\beta$ was decreased. This wave did not undergo any further bifurcations. Note, however, the presence of a disconnected branch of solutions, or an isola, in the system. These solutions were created in a saddle-node bifurcation, and correspond to amplitude vacillation and its subsequent period-doublings. Figure 19 shows an enlargement of the isola.

For $0.14<r<0.38$, the initially stable trivial solution bifurcated to a travelling wave as $\beta$ was decreased. This travelling wave then remained stable as $\beta$ was decreased to zero.

\subsection{Vertical phase tilt}

The phase difference, $\Theta$, determining the vertical phase tilt of the travelling wave solution, becomes less negative as $\beta$ increases. For example, when $r=0.25$ and $F=11.0, \Theta$ varies monotonically from $\sim-1.6$ to $\sim-1.8$ as $\beta$ increases from 0 to 6 . This implies that increasing $\beta$ suppresses the westward tilt with height of the instability. Physically, this reflects the fact that as $\beta$ increases, the velocity of the travelling wave increases. Consequently, there is an increase in the amount of the energy extracted from the mean-flow which is converted into the kinetic energy of the wave. Therefore, there is less potential energy available to support the westward tilt of the wave and so $\Theta$ decreases. This, in turn, reduces the ability of a growing disturbance to draw energy out of the mean-flow, thereby stabilizing the system. This stabilization effect can be seen in Eqs. (21) by noting that in the equations for $S, D$ and $X_{d}$, the nonlinear part contains a $\sin \Theta$ term. The decrease in the value of $\Theta$, therefore, causes a decrease in the strength of the nonlinear coupling in the dispersive system.

In addition to the vertical phase tilt of the travelling wave decreasing as $\beta$ increases, the nature of the amplitude vacillation also changes. Figure 20 shows the effect of increasing $\beta$ on the amplitude vacillation. It can be seen that, while the oscillation takes the form of a square wave on the $f$-plane, it appears more rounded as $\beta$ increases, i.e. the transfer of energy from the mean-flow to the wave (and vice versa) appears to be instantaneous on the $f$-plane, but becomes slower as $\beta$ is increased.

Thus, the period-doubling behavior observed in Pedlosky (1981a) is only typical of a small region of parameter space. Other sequences of bifurcations with decreasing $\beta$ can be found, depending on the strength of the dissipation present in the system.

The complete bifurcation behavior of the two-layer system in the $(r, \beta, F)$-parameter space is shown in Fig. 21. The true significance of the organizing center at $(r, \beta, F)=$ $\left(0,0, K^{2} / 2\right)$ can now be seen. It appears that the bifurcation 


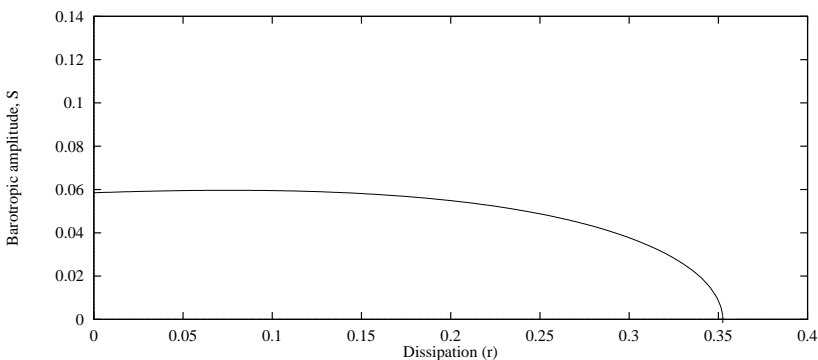

(a) $\beta=4.0$

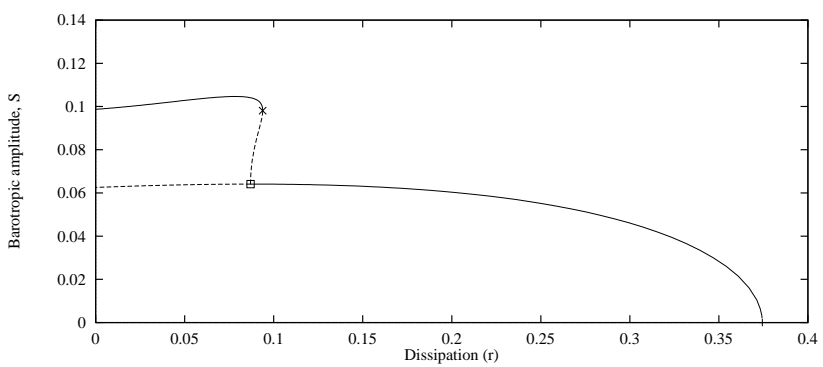

(c) $\beta=1.0$

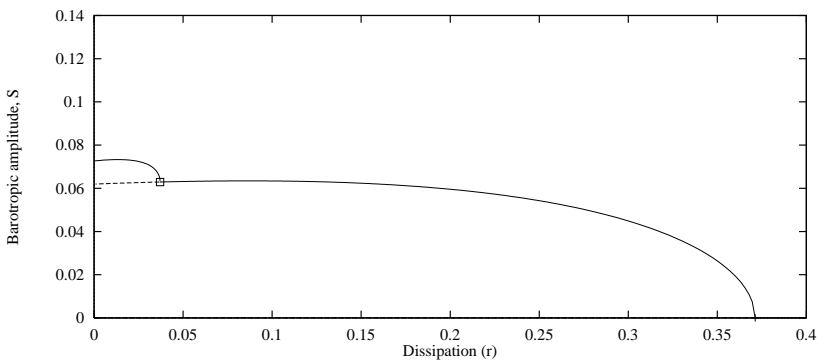

(b) $\beta=1.8$

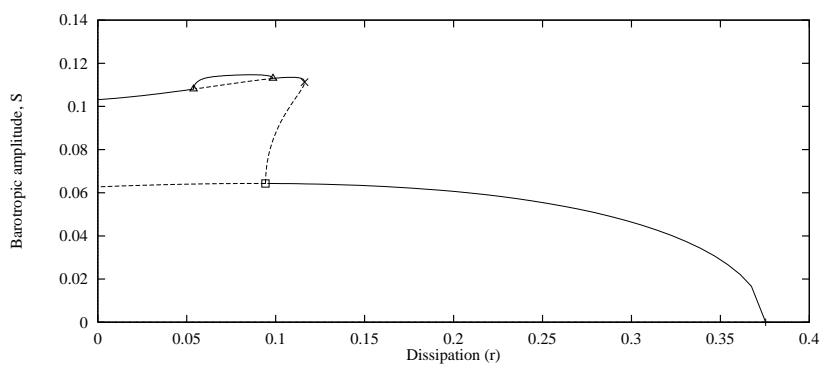

(d) $\beta=0.5$

Fig. 16. A series of one-parameter continuations, varying the dissipation parameter, $r$ at $F=11.0$, for different values of $\beta$.

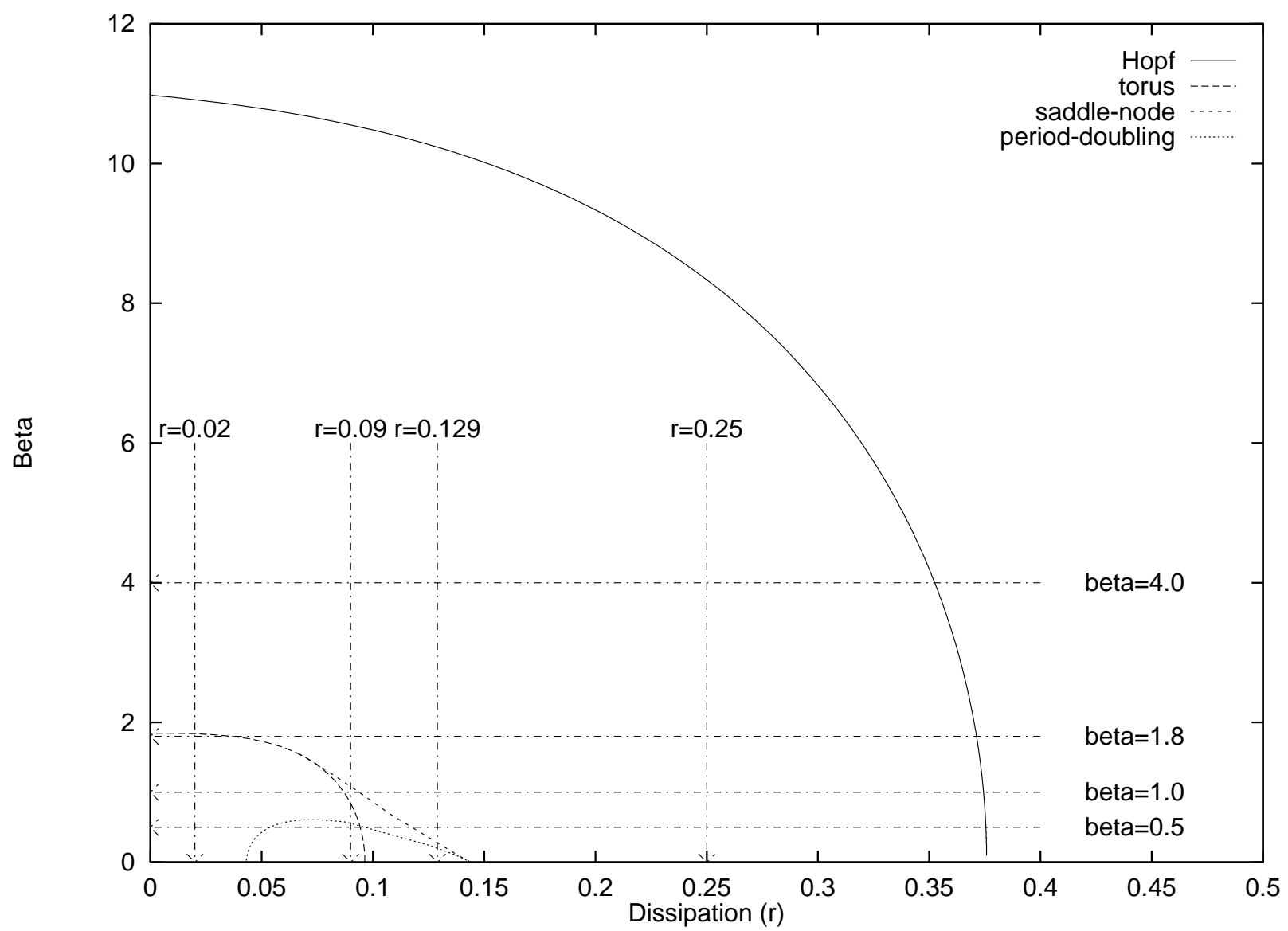

Fig. 17. The location on the $[r, \beta]$-plane of the one-parameter continuations shown in Fig. 16 and Fig. 18. 


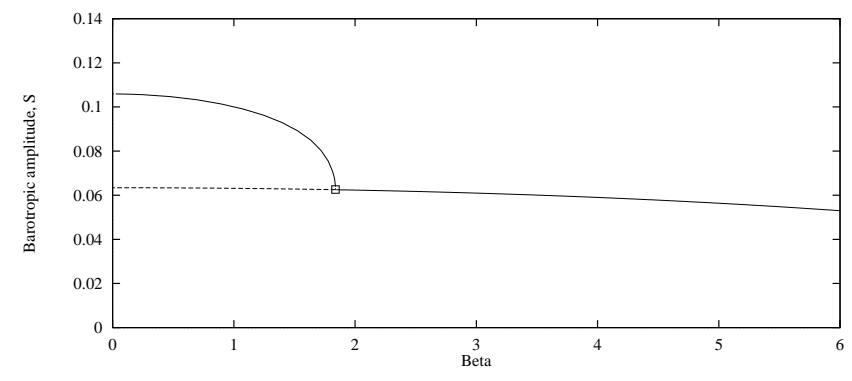

(a) $r=0.02$

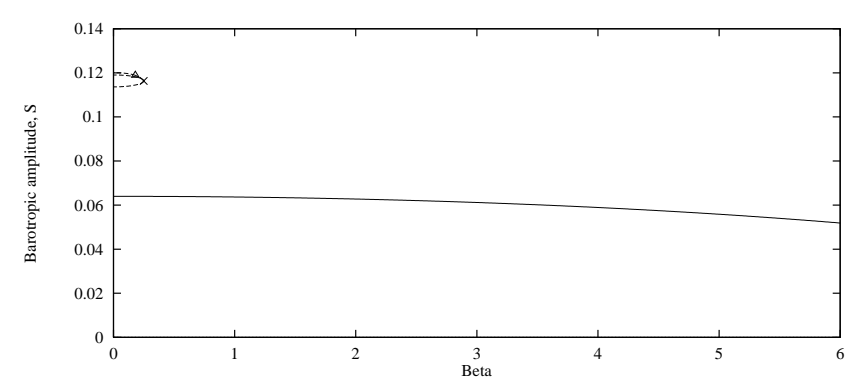

(c) $r=0.129$

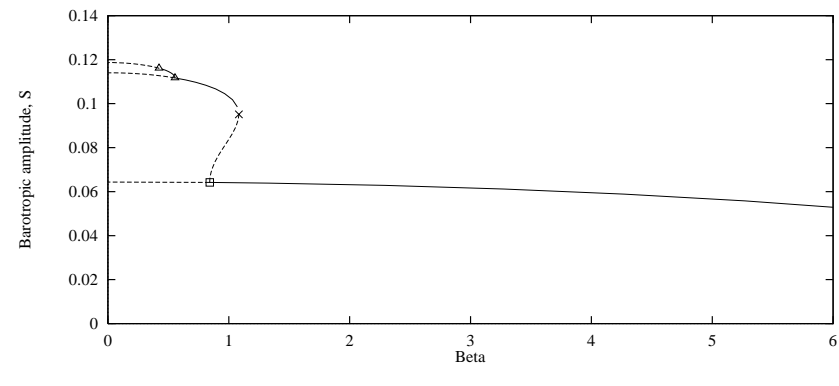

(b) $r=0.09$

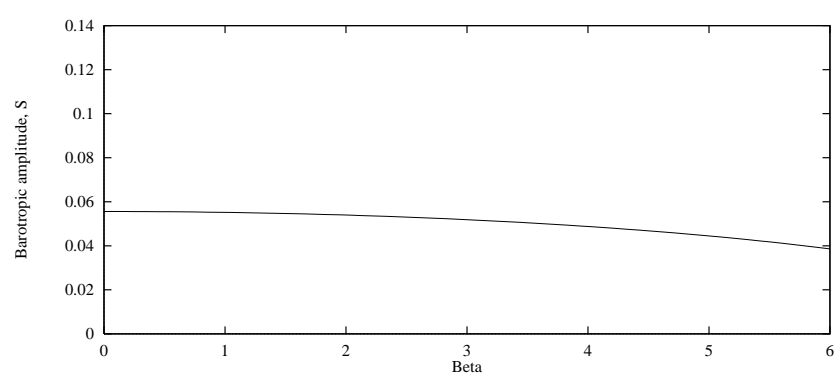

(d) $r=0.25$

Fig. 18. A series of one-parameter continuations, varying $\beta$ at $F=11.0$, for different values of the dissipation parameter, $r$.

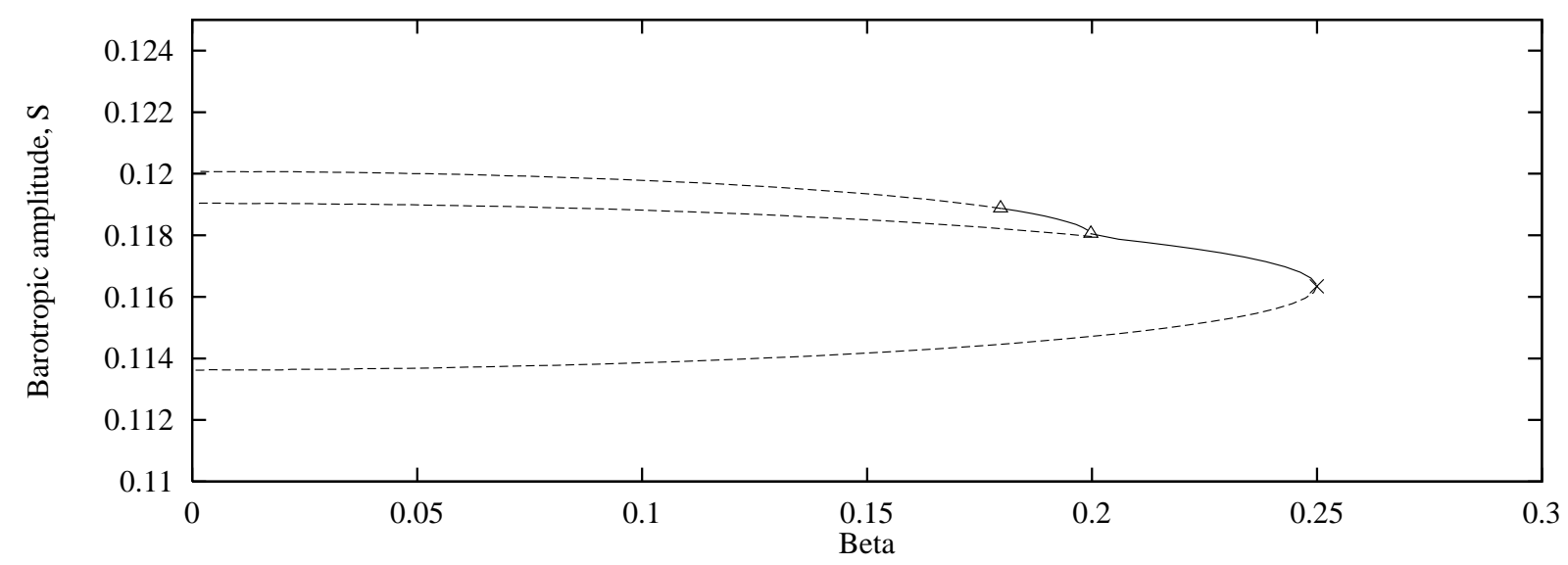

Fig. 19. An enlargement of the isolated solution branch shown in Fig. 18c.

structure of the two-layer system "originates" at this single degenerate bifurcation. Observe that, as $\beta$ is increased from zero, the $\beta$-plane Hopf bifurcation arises from the pitchfork of revolution on the $f$-plane; the subcritical torus bifurcation results from the subcritical Hopf bifurcation on the $f$-plane; and the saddle-node bifurcation is born from the homoclinic bifurcation on the $f$-plane. This is all in agreement with the symmetry arguments described in Sect. 4.2. Note that Fig. 21 also shows the curves derived by Pedlosky (1970) and Romea (1977), representing the onset of instability in the inviscid system.

\section{Bifurcations in the inviscid, weakly dispersive system}

In the weakly dispersive system, the first effect of dissipation is to destabilize the system. This is reflected in the wellknown discrepancy between the inviscid stability curve and the curve representing viscous stability in the limit $r \rightarrow 0$, in the full three-dimensional regime diagram of Fig. 21. Pedlosky (1981b) made the case for the inviscid curve being the "true" curve that marked the transition to instability, whereas Romea (1977) argued that the inviscid limit of the viscous theory was the "correct" curve. Romea based his argument on the fact that a slowly growing wave can be produced on a very long time scale in the region between the two curves 


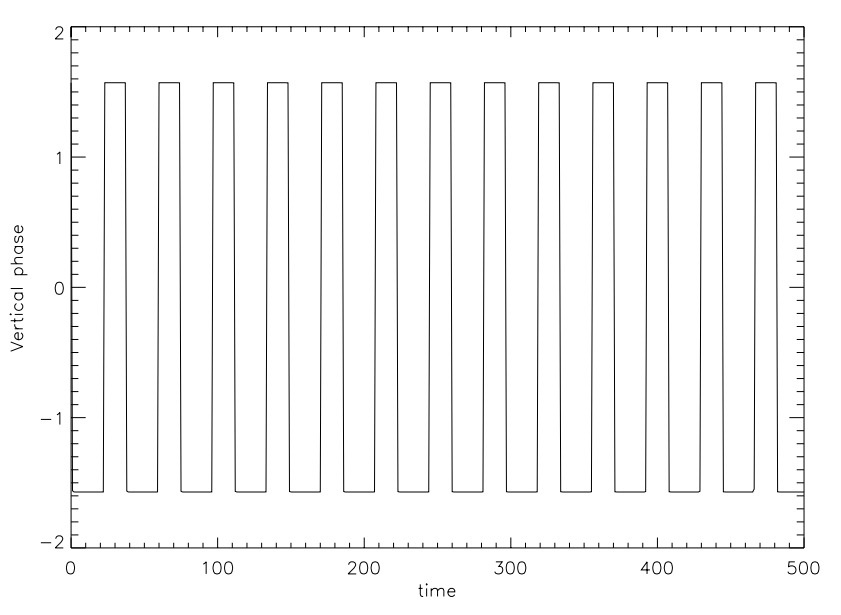

(a) $\beta=0(f$-plane $)$

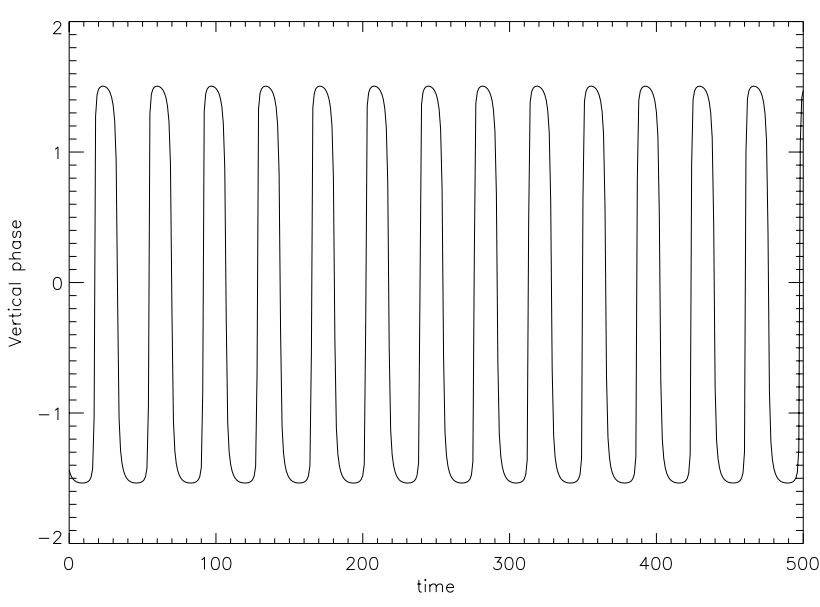

(b) $\beta=0.1$

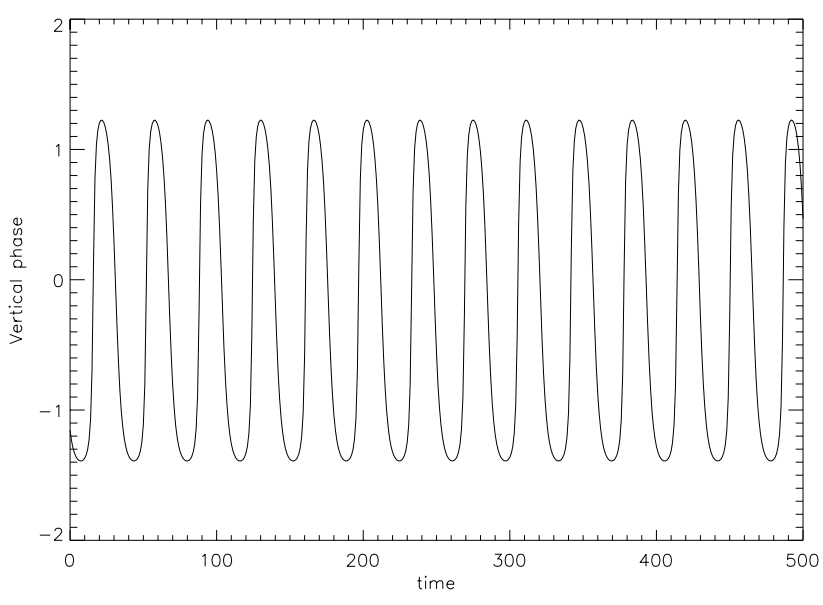

(c) $\beta=0.5$

Fig. 20. The vertical phase, $\Theta$, during an amplitude vacillation. As $\beta$ is increased, the response of the wave becomes slower and the oscillation appears more "rounded". as a result of nonlinear interactions. Neither Pedlosky nor Romea, however, gave detailed consideration to the type of bifurcations occurring on each curve. These bifurcations will now be discussed.

As has already been shown, the initial bifurcation in the viscous dispersive system is a Hopf bifurcation. In this bifurcation, two complex conjugate eigenvalues cross the imaginary axis, two other complex conjugate eigenvalues retain a real part that is less than zero, and one real eigenvalue is less than zero. In the inviscid $(\beta, F)$-plane, the system becomes Hamiltonian, i.e. the eigenvalues are either always purely imaginary or are equal with opposite signs. The inviscid system is neutrally stable and is part of the center-manifold of the degenerate bifurcation at $(\beta, F)=\left(0, K^{2} / 2\right)$ and the Hartman-Grobman theorem (see e.g. Guckenheimer and Holmes, 1983) no longer applies. Nonlinear terms must, therefore, be taken into account when analysing the stability of the system. Hence, Romea's argument that, on the $r=0$ plane, instability actually results from nonlinear interactions is probably correct and it follows that the inviscid limit of the viscous theory is probably the correct marker for the onset of instability. While Romea's work took nonlinear terms into account, Pedlosky's inviscid analysis concentrated on looking for the transition to linear instability. This occurs in a bifurcation specific to Hamiltonian systems that is illustrated in Fig. 22.

For small values of $F$, all of the eigenvalues are situated on the imaginary axis and there are two distinct pairs of imaginary eigenvalues and one zero eigenvalue. As $F$ is increased, the imaginary pairs approach each other until, at a critical value, the pairs become identical and we have a case of $1: 1$ resonance. For values of $F$ larger than this critical value, the two complex pairs are no longer purely imaginary. Instead they contain a real part. This bifurcation mechanism is known as a Trojan or Hamiltonian Hopf bifurcation (see Bridges, 1990; Woods and Champeneys, 1999; Champeneys, 1999).

In the viscous, dispersive two-layer system, the Trojan bifurcation mechanism is not applicable since the requirement that the real part of two pairs of complex eigenvalues must be zero is never satisfied. Comparing this special inviscid bifurcation with the Hopf bifurcation present in the viscous system, therefore, does not make sense, since the two instability mechanisms are different. This further strengthens Romea's claim that the inviscid limit of the viscous problem is the correct limit to use when trying to determine the onset of instability in the two-layer system.

\section{Conclusions}

In this paper, we have extended the analysis of Part I to perform for the first time a detailed examination of bifurcations present in the two-layer $\beta$-plane spectral amplitude equations. It was shown that the behavior predicted by various multiple-scales approximations is reproduced by the spectral amplitude equations. In particular, it was shown that for 


\section{$\mathrm{SO}(2)$ symmetry half-space}

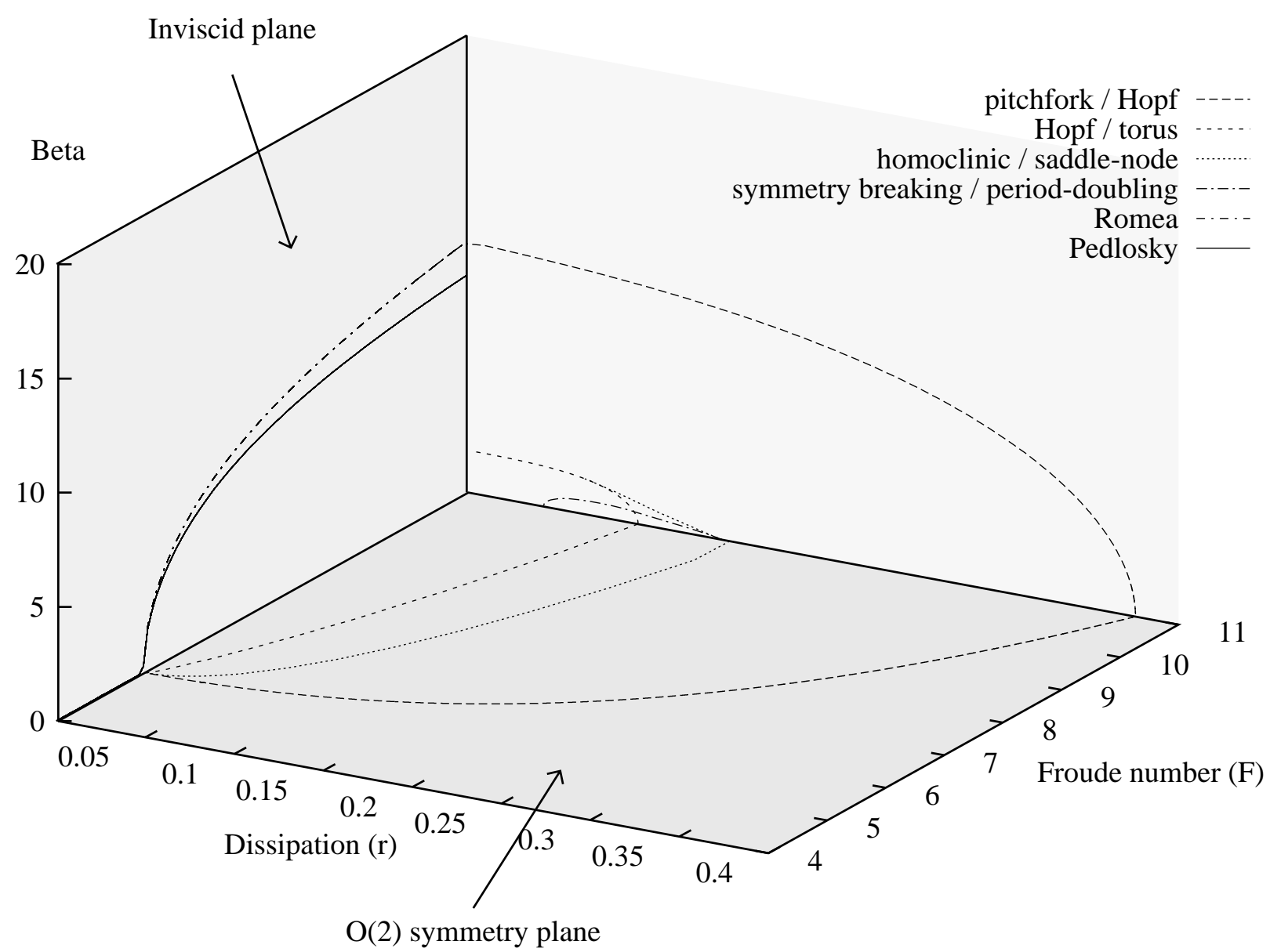

Fig. 21. The bifurcations of the two-layer model in the $[r, \beta, F]$ parameter space. Note that the pitchfork, homoclinic and Hopf bifurcations found on the $f$-plane change to Hopf, saddle-node and torus bifurcations as $\beta$ becomes non-zero. For completeness, the two instability curves derived by Pedlosky (1970) and Romea (1977) for the inviscid system are shown on the $r=0$ plane. Observe that all the bifurcations appear to emerge from the organizing center at $(r, \beta, F)=\left(0,0, K^{2} / 2\right)$.

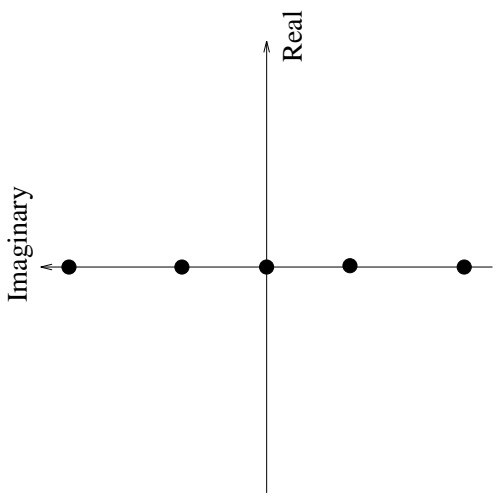

(a)

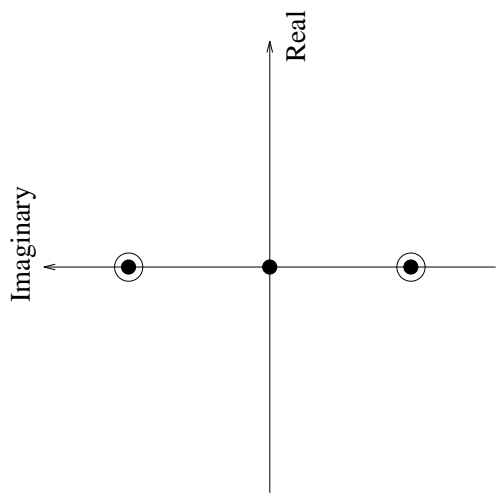

(b)

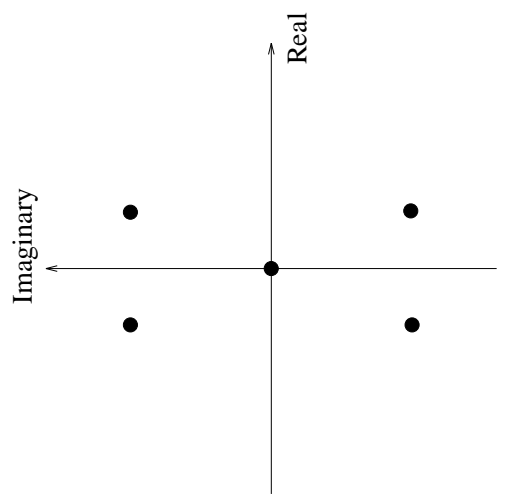

(c)

Fig. 22. A Trojan bifurcation in the complex plane. Two pairs of imaginary eigenvalues, (a), move into one to one resonance, (b), causing linear instability as two complex-conjugate eigenvalues are produced (c). 
small values of $\beta$ the spectral amplitude equations reproduce the behavior associated with the complex Lorenz equations.

Using AUTO86 we performed a more detailed bifurcation analysis of the weakly dispersive regime than that of Fowler and McGuinness (1984) and support their conclusion that the weakly dispersive system contains a subcritical torus bifurcation, with the solutions created in this bifurcation turning back on themselves in a saddle-node bifurcation. The results of this study, however, disagreed with their suggestion that a homoclinic connection is present in the system and is responsible for the creation of period-doubling cascades. Indeed, our study showed that the $S O$ (2) symmetry of the $\beta$ plane destroyed the homoclinic connection observed in the $f$-plane system, and created a saddle-node bifurcation instead. The dynamics of the $\beta$-plane system are, therefore, fundamentally different from those of the $f$-plane model. Consequently, the $f$-plane approximation may not simply be viewed as the $\beta \rightarrow 0$ limit of the $\beta$-plane approximation.

Moreover, a strong resonant response was found along some branches of period-doubled tori and was repeated for successive branches of period-doubled tori. Furthermore, this investigation showed that, in the weakly dispersive regime, the spectral amplitude equations exhibit frequencylocking within narrow tongues emanating from various cusp bifurcations. We argued that this frequency-locking was probably a result of circle map dynamics, in contrast to the work of Fowler and McGuinness who found no such frequency-locking in their weakly-dispersive multiple-scales approximation.

Pedlosky's observation that a cascade of inverse perioddoubling bifurcations occurred with increasing $\beta$ was also verified and we subsequently showed other bifurcation sequences to be possible, by increasing $\beta$ at different values of the dissipation $r$. These sequences included a disconnected branch of period-doubling bifurcations at large dissipation.

The analyses of Pedlosky (1970) and Romea (1977) of the inviscid bifurcation problem in the dispersive regime were then compared. It was concluded that Pedlosky's study relied on an instability mechanism specific to Hamiltonian systems, whereas the mechanism of Romea was common to both viscous and inviscid systems. Consequently, any comparison between viscous and inviscid instability should be based on Romea's analysis.

Finally, a three-dimensional regime diagram showing behavior as a function of $r, \beta$ and $F$ were constructed. This allowed for the true importance of the degenerate bifurcation at $(r, \beta, F)=\left(0,0, \frac{K^{2}}{2}\right)$, as an organizing center, to be seen.

The extent to which such a highly truncated model as presented here and in Lovegrove et al. (2001) provides an appropriate description of the bifurcation behavior in a physical system (such as in laboratory experiments or even a planetary atmosphere) is an important issue, though a detailed discussion is beyond the immediate scope of this paper. Some general features of the transition to geostrophic chaos and turbulence in simple models have been reviewed by Klein (1990), in which some aspects of the generic problems are raised concerning the possible lack of robustness of the behavior of spatially truncated models as the level of truncation is relaxed (cf Curry et al. (1984) for a comparable problem in fluid dynamics).

It has long been observed (e.g. Hide and Mason, 1975; Hart, 1979) that the initial bifurcations from axisymmetric flow at weak-moderate supercriticality in baroclinic systems studied in the laboratory entail the emergence of regimes in which the flow is dominated by just one (or a small number of) wave mode(s), with either steady or periodically-varying amplitude or structure. Such a regime is highly reminiscent of the present model, with or without a $\beta$-effect. At higher supercriticality, experiments (e.g. Hart, 1985; Ohlsen and Hart, 1989; Read et al., 1992; Früh and Read, 1997) have shown evidence for several alternative routes culminating in low-dimensional chaos, but which include intermediate quasi-periodic states and/or period-doubling cascades starting from periodically vacillating waves, with similarities in at least some cases to the bifurcation sequences found in the present models.

As found for the $\beta$-plane QG spectral amplitude equation models presented above, departures from spatial $O(2)$ symmetries may be important in this regard. Mundt and Hart (1994), for example, found that the spatial complexity of bifurcations increased sharply with supercriticality in their numerical study of transitions to baroclinic chaos in a twolayer, zonally-periodic rectangular channel, even though the temporal behavior still involved bifurcations via periodic and quasi-periodic states. In a comparable study in cylindrical geometry, however, where the $\mathrm{O}(2)$ reflectional symmetry was broken, the spatial structure of baroclinic instabilities tended to remain much simpler (Mundt et al., 1995), in generally good agreement with experiments (Hart, 1985; Ohlsen and Hart, 1989; Read et al., 1992; Früh and Read, 1997) and thereby retaining many features of the present models well into the supercritical regime.

In conclusion, we note that such spatial simplicity in moderately supercritical baroclinic instability is not confined to small-scale laboratory systems, but might also be a feature of large-scale atmospheric flows under certain conditions (such as those prevailing on planets such as Mars). Collins and James (1995), for example, showed evidence for the development of regular, near-steady baroclinic waves in a simplified, high resolution, large-scale atmospheric general circulation model run under conditions intended to emulate those in the Martian atmosphere. Such results were later confirmed in a much more sophisticated and realistic atmospheric model of the Martian atmosphere by Collins et al. (1996); Read et al. (1998), together with evidence from analyses of data from the Viking Lander spacecraft for low-dimensional chaotic motion on Mars itself. While it is highly likely that the spatial complexity of baroclinic flows eventually diverges strongly from that assumed in the present models, given sufficient supercriticality, there would seem to be substantial evidence to suggest that idealized studies using low-dimensional systems, such as presented here, can provide valuable insights into the nonlinear dynamics of a rich variety of physical sys- 
tems.

\section{Appendix A Coefficients of the single-wave model}

The coefficients to Eqs. (11) are:

$$
\begin{aligned}
& K^{2}=k_{1}^{2}+l_{1}^{2} \\
& \Delta_{s}=r\left[1+r \epsilon K^{2}\right] \\
& \Delta_{d}=\frac{r K^{2}}{\left(K^{2}+2 F\right)}\left[1+r \epsilon\left(K^{2}+2 F\right)\right] \\
& \bar{\Delta}=\frac{r l_{1}^{2}}{\left(l_{1}^{2}+2 F\right)}\left[1+r \epsilon\left(l_{1}^{2}+2 F\right)\right] \\
& \beta_{s}=\left[\frac{\beta}{K^{2}}-U_{s}\right] k_{1} \\
& \beta_{d}=\left[\frac{\beta}{\left(K^{2}+2 F\right)}-U_{s}\right] k_{1} \\
& v_{s}=U_{d} k_{1} \\
& v_{d}=U_{d} \frac{\left(K^{2}-2 F\right)}{\left(K^{2}+2 F\right)} k_{1} \\
& \gamma_{s}=\frac{16 k_{1}^{3}}{6 K^{2}} \\
& \gamma_{d}=\frac{16 k_{1}\left(k_{1}^{2}-2 F\right)}{6\left(K^{2}+2 F\right)} \\
& \bar{\gamma}=\frac{32 F k_{1}}{3\left(l_{1}^{2}+2 F\right)} .
\end{aligned}
$$

All the numerical continuation computations in this paper were carried out using the 1986 version of the widely available package AUTO Doedel (1981); Doedel and Kernevez (1986). Some codes written to produce the figures in this paper, together with lists of parameters, will be made available via the website http://www.atm.ox.ac.uk/users/read/LMRpaper2.html.

Acknowledgements. AFL acknowledges support under a research studentship from the UK Engineering and Physical Sciences Research Council and Smiths System Engineering. We are grateful to the anonymous referees for comments and suggestions which led to significant improvements to this version of the paper.

\section{References}

Arnol'd, V. I.: Small denominators, i: Mappings of the circumference into itself, AMS Transl. Series 2, 46, 213-284, 1965.

Block, L.: Periods of periodic points of maps of the circle which have a fixed point, Proc. Amer. Math. Soc., 82, 481-486, 1981.

Bridges, T. J.: Bifurcation of periodic solutions near a collision of eigenvalues of oppposite signature, Math. Proc. Camb. Phil. Soc., 108, 575-601, 1990.

Brindley, J. and Moroz, I. M.: Lorenz attractor behavior in a continuously stratified baroclinic fluid, Phys. Lett., 77A, 441-444, 1980.
Champeneys, A. R.: Homoclinic orbits in reversible systems II: multi-bumps and saddle-centres, CWI Quarterly, 12, 185-212, 1999.

Chossat, P.: Forced reflectional symmetry breaking of an $O$ (2)symmetric homoclinic cycle, Nonlinearity, 6, 723-731, 1993.

Collins, M. and James, I. N.: Regular baroclinic transient waves in a simplified global circulation model of the martian atmosphere, J. Geophys. Res., 100, 14421-14 432, 1995.

Collins, M., Lewis, S. R., Read, P. L., and Hourdin, F.: Baroclinic wave transitions in the Martian atmosphere, Icarus, 120, 344357, 1996.

Curry, J. H., Herring, J. H., Loncaric, J., and Orszag, S. A.: Order and disorder in two- and three-dimensional Bénard convection, J. Fluid Mech., 147, 1-38, 1984.

Doedel, E.: Auto: a program for the automatic bifurcation analysis of autonomous systems, Cong. Numer., 30, 265-284, 1981.

Doedel, E. J. and Kernevez, J. P.: Auto: software for continuation and bifurcation problems in ordinary differential equations, Tech. rep., Applied Mathematics Report, California Institute of Technology, 1986.

Drazin, P. G.: Nonlinear Systems, vol. 10 of Cambridge Texts in Applied Mathematics, Cambridge University Press, Cambridge, 1992.

Fowler, A. C. and McGuinness, M. J.: On the nature of the torus in the complex Lorenz equations, SIAM J. Appl. Math., pp. 681700, 1984.

Fowler, A. C., Gibbon, J. D., and McGuinness, M. J.: The complex Lorenz equations, Physica D, 4, 139-163, 1982.

Fowler, A. C., Gibbon, J. D., and McGuinness, M. J.: The real and complex Lorenz equations and their relevance to physical systems, Physica D, 7, 126-134, 1983.

Früh, W.-G. and Read, P. L.: Wave interactions and the transition to chaos of baroclinic waves in a thermally driven rotating annulus, Phil. Trans. Roy. Soc. Lond, 355, 101-153, 1997.

Gibbon, J. D. and McGuinness, M. J.: The real and complex lorenz equations in rotating fluids and lasers, Physica D, 5, 108-122, 1982.

Guckenheimer, J. and Holmes, P. J.: Nonlinear Oscillations, Dynamical Systems, and Bifurcations of Vector Fields, SpringerVerlag, New York, Berlin, Heidelberg, Tokyo, 1983.

Hart, J. E.: Finite amplitude baroclinic instability, Ann. Rev. Fluid Mech., 11, 147-172, 1979.

Hart, J. E.: A laboratory study of baroclinic chaos on the $f$-plane, Tellus, 37 A, 286-296, 1985.

Hidalgo, M. C.: Periods of periodic points for transitive degree one maps of the circle with a fixed point, Acta Math. Univ. Comenianae, LXI(1), 11-19, 1992.

Hide, R. and Mason, P. J.: Sloping convection in a rotating fluid, Advances in Physics, 24, 47-99, 1975.

Holopainen, E. O.: On the effect of friction in baroclinic waves, Tellus, 13, 363-367, 1961.

Klein, P.: Transitions to chaos in unstable baroclinic systems: a review, Fluid Dyn. Res., 5, 235-254, 1990.

Knobloch, E.: Symmetry and instability in rotating hydrodynamic and magnetohydrodynamic flows, Phys. Fluids, 8, 1446-1454, 1996.

Li, T.-Y. and Yorke, J. A.: Period three implies chaos, Amer. Math. Monthly, 82, 985-992, 1975.

Lovegrove, A. F. L.: Bifurcations and instabilities in rotating twolayer fluids, Ph.D. thesis, University of Oxford, 1998.

Lovegrove, A. F. L., Moroz, I. M., and Read, P. L.: Bifurcations and instabilities in rotating, two-layer fluids: I. $f$-plane, Nonlin. 
Proc. Geophys., 8, 21-36, 2001.

Mackay, R. S. and Tressier, C.: Transition to topological chaos for circle maps, Physica D, 19, 206-237, 1986.

Marek, M. and Schreiber, I.: Chaotic behavior of deterministic dissipative systems, Cambridge University Press, Cambridge, 1991.

Moroz, I. M.: Slowly varying baroclinic waves in dispersive and dissipative systems, Ph.D. thesis, University of Leeds, 1981.

Mundt, M. D. and Hart, J. E.: Secondary instability, EOF reduction and the transition to baroclinic chaos, Physica D, 78, 65-92, 1994.

Mundt, M. D., Brummell, N. H., and Hart, J. E.: Linear and nonlinear baroclinic instability with rigid sidewalls, J. Fluid Mech., 291, 109-138, 1995.

Newell, A. C.: The post bifurcation stage of baroclinic instability, J. Atmos. Sci., 29, 64-76, 1972.

Ohlsen, D. R. and Hart, J. E.: Transitions to baroclinic chaos on the $\beta$-plane, J. Fluid Mech., 203, 23-50, 1989.

Pedlosky, J.: An initial value problem in the theory of baroclinic instability, Tellus, 16, 12-17, 1964.

Pedlosky, J.: Finite-amplitude baroclinic waves, J. Atmos. Sci., 27, 15-30, 1970.

Pedlosky, J.: Finite-amplitude baroclinic waves with small dissipation, J. Atmos. Sci., 28, 587-597, 1971.

Pedlosky, J.: The nonlinear dynamics of baroclinic wave ensembles, J. Fluid Mech., 102, 169-209, 1981a.
Pedlosky, J.: The effect of $\beta$ on chaotic behavior of unstable baroclinic waves, J. Atmos. Sci., 38, 717-731, 1981b.

Read, P. L., Bell, M. J., Johnson, D. W., and Small, R. M.: Quasiperiodic and chaotic flow regimes in a thermally-driven, rotating fluid annulus, J. Fluid Mech., 238, 599-632, 1992.

Read, P. L., Collins, M., Früh, W.-G., Lewis, S. R., and Lovegrove, A. F.: Wave interactions and baroclinic chaos: a paradigm for long timescale variability in planetary atmospheres, Chaos, Solitons \& Fractals, 9, 231-249, 1998.

Romea, R. D.: The effects of friction and $\beta$ on finite-amplitude baroclinic waves, J. Atmos. Sci., 1689-1695, 1977.

Sarkovskii, D. H.: Coexistence of cycles of a continuous map of a line into itself, Ukr. Math. Z., 16, 61-71, 1964.

Swift, J. W. and Wiesenfeld, K.: Supression of period doubling in symmetric systems, Phys. Rev. Lett., 52, 705-708, 1984.

Weng, H.-Y., Barcilon, A., and Magnan, J.: Transitions between baroclinic flow regimes, J. Atmos. Sci., 43, 1760-1777, 1986.

Wiggins, S.: Introduction to Applied Nonlinear Dynamical Systems and Chaos, Springer-Verlag, New York, 1990.

Wolf, A., Swift, J. B., Swinney, H. L., and Vastano, J. A.: Determining Lyapunov exponents from a time series, Physica D, 16, 285-317, 1985.

Woods, P. D. and Champeneys, A. R.: Heteroclinic tangles and homoclinic snaking in the unfolding of a degenerate reversible Hamiltonian Hopf bifurcation, Physica D, 129, 147-170, 1999. 